\title{
KNOWING What OThers KnOW: Coordination Motives in Information ACQUisition
}

\author{
Christian HellwiG* \\ University of California, Los Angeles \\ and CEPR
}

\author{
LAURA VELDKAMP \\ New York University \\ Stern School of Business
}

March 12, 2008

\begin{abstract}
We explore how optimal information choices change the predictions of strategic models. When a large number of agents play a game with strategic complementarity, information choices exhibit complementarity as well: If an agent wants to do what others do, they want to know what others know. This makes heterogeneous beliefs difficult to sustain and may generate multiple equilibria. In models with substitutability, agents prefer to differentiate their information choices. We use these theoretical results to examine the role of information choice in recent price-setting models and to propose modeling techniques that ensure equilibrium uniqueness.
\end{abstract}

\footnotetext{
*We thank Andy Atkeson, Willie Fuchs, Ricardo Reis, Aleh Tsyvinski, Xavier Vives, Ivan Werning, and seminar participants at Bonn, CalTech, Chicago, Columbia, Iowa, Princeton, Purdue, UCLA, UCSD the 2005 AEA meetings, the Cowles Workshop on Coordination Games, 2006 EEA meetings, 2006 North American meetings of the Econometric Society, 2006 Philadelphia macro meetings, and 2006 SED meetings for helpful comments. We also thank two anonymous referees and our editor whose comments helped to substantially improve the paper. Keywords: Coordination games, information choice, beauty contest game, price-setting. JEL codes: D83, C72, E3.
} 
Recent work in many fields, including nominal price adjustment, asset pricing, financial crises, political economy or competition in networks, shows that equilibrium outcomes, welfare and determinacy all depend on what information agents can observe. ${ }^{1}$ In most of these models, the information that agents are endowed with is exogenous. It usually represents a guess by the economist about what information is available. In practice, the information people observe depends both on its availability and on their choice of what to learn.

We ask what agents choose to observe when information is costly, and how these information choices change the equilibrium outcomes. Comparing games with and without information acquisition, we highlight one similarity and one difference. Our main result focuses on the similarity: When agents choose how much information to acquire before choosing strategic actions, the information choice inherits the same strategic motives as the actions. Agents who want to do what others do want to know what others know. Decision complementarities in the underlying game produce coordination motives in information acquisition. The converse is also true. Agents who want to do what others do not, want information others do not know. The difference is that complementarity in information acquisition can cause the number of equilibria to change. Adding information choice can resurrect multiple equilibria, even if the exogenous information model has one equilibrium. Whether the equilibrium is unique depends on the public or private nature of the information agents can acquire.

To explore strategic settings, we use a version of Morris and Shin's (2002) static 'beauty contest' game (section 1). In a beauty contest, agents' payoffs depend on how closely their action matches an unknown target that depends on an exogenous state and other agents' actions. This game has been used to describe many strategic settings with incomplete information, including: financial markets, investment decisions, or price adjustment with monopolistic competition. We add an initial stage, in which agents can improve the quality of their information, at a cost.

It is well-known that common information helps agents to coordinate. Our main result goes beyond this existing intuition in two ways. First, when actions are complements, information

\footnotetext{
${ }^{1}$ Among many other papers, see Mankiw and Reis (2002), Woodford (2002), Lorenzoni (2006) or Zeira (1999) for models of price adjustment and business cycles. See Veronesi (1999), Bikhchandani, Hirshleifer, and Welch (1992), Caplin and Leahy (1994), Morris and Shin (1998), Goldstein and Pauzner (2005), Angeletos and Werning (2006), or Hellwig, Mukherji, and Tsyvinski (2005) for models of financial crises. See Farrell and Klemperer (2007), Stromberg (2001), or Edmond (2005) for models of network competition and political economy.
} 
acquisition is complementary, even if that information is a private signal, conditionally independent from all other signals. Second, one might think that agents whose actions are substitutes would still want to know what others know, to better take the opposite action. This is not the case; agents want to know what others do not. Section 1.2 shows that the strategic motives in information acquisition come from the effects that others' information choices have on the covariance between the unobserved state and the aggregate action. When others are well-informed, aggregate actions covary highly with the state. If actions are complements, this covariance makes learning about the true state even more valuable because it reveals information about others' actions. But when actions are substitutes, the aggregate actions and the state have offsetting effects on a firm's optimal decision: Knowing the value of the state is high encourages a high action, while knowing that others will choose high actions encourages a low action. Thus, the optimal action varies less across states, when others are informed. If the state matters less for decisions, knowing the state becomes less valuable. In short, information loses value when other firms observe it.

Section 1.3 shows that complementarity in actions, combined with investment in potentially public information, is a new source of multiple equilibria: If an agent purchases one more bit of potentially common information than any other agent, this bit remains private, and its value is the marginal value of private information. But if the agent considers not purchasing a bit of information that is observed by all others, its value is the marginal value of public information. This discontinuity in marginal value arises because potentially public information only has the value of public information when others choose to observe it. The result is that when actions are complementary, agents want to observe additional information, only if others do so as well. Equilibrium public information choice is indeterminate, even though the same model, with any of the feasible information choices exogenously imposed, would have a unique equilibrium.

For applied theorists who want to formulate a model with coordination motives and information choice and who want a unique prediction from that model, the solution lies in private information. When agents invest only in conditionally independent, private signals, complementarity is weak. The result is a unique equilibrium. While endowing agents with heterogeneous information guarantees an unique equilibrium in Morris and Shin (2002), the game with information choice imposes an additional requirement for equilibrium uniqueness: The information agents choose to acquire must 
also be private. Unfortunately, a small amount of private noise added to agents' chosen signals will not suffice. For the equilibrium to be unique, signal noise across agents must be independent. But such an independence assumption should be well-founded because it is stringent and it ultimately determines the model's predictions.

Section 2 uses the theoretical results from the preceding section to critically examine recent price-setting models with information choice. Woodford (2002) and Mankiw and Reis (2002) add exogenous information heterogeneity and price-setting complementarity to get bigger real effects from a demand shock in a setting like that of Lucas (1972). Sims (2003), Moscarini (2004) and Reis (2006) model information choice, but neglect its strategic nature. Following Reis (2006), we analyze monopolistically competitive firms who adjust prices every period, but update their information infrequently. In each period, firms choose whether or not to pay a cost to "plan." A firm that plans observes all shocks and prices realized since it last planned.

Because competitive monopolists have a coordination motive when setting prices, they also have a coordination motive in choosing when to plan. Planning complementarity changes the predictions of the exogenous information and non-strategic learning models. Firms acquire less information about demand because other firms are imperfectly informed. When all firms know less about demand, average prices covary less with it. This price-demand covariance is the inverse of price stickiness. Thus planning complementarity plays an important role in generating a realistic amount of price stickiness, which is what the price-setting models are designed to explain.

Complementarity is both a blessing and a curse. Planning complementarity creates two problems that follow from the two main results we establish in section 1. First, because firms choose a date at which to plan, they may synchronize their information acquisition by planning all on the same date. This result is the dynamic analog to the static result that all firms want to observe the same signals. Synchronized planning fundamentally alters the price response to a monetary shock. Instead of the gradual price adjustment predicted by the non-strategic models, synchronizedplanning prices do not react at all, until they jump from the burst of new information. Second, multiple equilibria can arise because of the public nature of price-relevant information (section 2.3). Multiplicity makes the rate of price adjustment, crucial for monetary policy analysis, indeterminate. Section 2.4 offers a solution; requiring the information price-setters acquire to be private 
can deliver a unique equilibrium. One micro-founded theory that justifies such an assumption is rational inattention (Sims 2003). Agents with limited channel capacity always observe signals that are the true state, plus independent noise.

These results provide a way to evaluate information assumptions made in a variety of strategic settings. In settings with substitutability, such as financial investment, agents have a strong incentive to differentiate their information. Markets with common knowledge, usually studied in finance, are unlikely to arise. In models with complementarities, such as monopolistic price-setting, agents want to acquire the same information. Heterogeneous beliefs are difficult to sustain endogenously. In price-setting models, this effect creates the paradox that the pricing complementarity needed to slow down price adjustment also makes differences in information and a gradual adjustment of the aggregate price level difficult to sustain.

\section{A Beauty Contest Model with Information Choice}

We convey our main intuition using a beauty contest game (as in Morris and Shin (2002)). Agents are rewarded for taking actions close to two values: the value of the true state and the average action of other agents. ${ }^{2}$ Agents choose how precise a signal to purchase about the true state, before they play this game. When other firms are poorly informed, signals reveal mostly just information about the state. When others are well-informed, signals are informative about aggregate actions as well. The results also illustrate how information choices can affect macroeconomic outcomes. When agents learn more, aggregate actions become more volatile, and more correlated with the true state. By quadratically approximating objectives, we can map many models into this linearquadratic framework. Section 2 is an example of such an approximation.

\section{$1.1 \quad$ Model description}

In stage one, nature draws the state variable $s$ and a series of signals about $s$. Agents choose which of these signals to observe. In stage two, agents observe their chosen signals and pick an optimal

\footnotetext{
${ }^{2}$ Other variants of the beauty contest game have been used by Allen, Morris, and Shin (2006) to study asset pricing, by Froot, Scharfstein, and Stein (1992) to study the incentives of financial analysts, and by Angeletos and Pavan (2007a), Hellwig (2006) and Cornand and Heinemann (2007) to study the welfare effects of information provision.
} 
action. Agents choose actions simultaneously. We'll call this action, setting the price $p$. The results generalize to a range of strategic actions in a quadratic loss setting.

A measure one continuum of agents, indexed by $i \in[0,1]$ choose a price $p_{i} \in \mathbb{R}$ to minimize the squared distance between their price and an unknown target price $p^{*}$. The target price has a component that depends on an unknown state $s$ and a component that depends on the aggregate price: $\bar{p}=\int p_{i} d i$ :

$$
u\left(p_{i}, \bar{p}, s\right)=-\frac{1}{(1-r)^{2}}\left(p_{i}-p^{*}\right)^{2} \quad \text { where } p^{*}=(1-r) s+r \bar{p}
$$

If $s$ is common knowledge, the best response is $p_{i}=(1-r) s+r \bar{p}$, and $p_{i}=\bar{p}=s$ constitutes the unique equilibrium. The coefficient $r<1$ measures the complementarity/substitutability of agents' decisions. If $r>0$, decisions are complementary: Best responses are increasing in the prices set by other agents. if $r<0$, decisions are strategic substitutes. A higher $r$ means more complementarity. The normalization by $(1-r)^{2}$ is for pure notational convenience. We will use lower-case variables in regular type face for scalars, boldface lower-case for vectors, and capital letters for matrices.

Information choices Nature selects a state $s \sim \mathcal{N}(0,1)$. In addition, nature selects a $k \times 1$ vector of common signal noises $\mathbf{u} \sim \mathcal{N}\left(0, I_{k}\right)$, which are independent of the state $s$. Finally, for each agent, nature selects an $l \times 1$ vector of idiosyncratic signal noises $\mathbf{v}^{i}$, which are independently and identically distributed across agents, with distribution $\mathbf{v}^{i} \sim \mathcal{N}\left(0, I_{l}\right)$, and are independent of $s$ and $\mathbf{u}$.

These shocks generate an $n \times 1$ vector of potentially observable signals $\mathbf{z}^{i}$ :

$$
\mathbf{z}^{i}=\mathbf{1}_{n} \cdot s+A \mathbf{u}+B \mathbf{v}^{i}
$$

$\mathbf{1}_{n}$ is an $n \times 1$ vector of ones and $A$ and $B$ are arbitrary $(n \times k)$ and $(n \times l)$ matrices of coefficients. The matrix $[A B]$ has row rank $n$, so that no subset of signals in $\mathbf{z}^{i}$ can fully reveal $s$.

This formulation imposes as little restriction as necessary on the possible information structure. In particular, by setting either $A$ or $B$ equal to zero, we can allow for special cases where all signals are either purely private, i.e. with noise independent across agents, or common. We also allow for 
an arbitrary structure of correlation across agents and/or signals.

Agents choose to observe a subset of the signal vector $\mathbf{z}^{i}$. Formally, their information choice consists of a vector $\chi^{i}$ comprised of zeros and ones, such that $\chi_{j}^{i}=1$ means that agent $i$ observes the $j^{\text {th }}$ entry of $\mathbf{z}^{i}$. The information choice $\chi^{i}$ comes from a compact feasible set $\Upsilon \subseteq\{0,1\}^{n}$. The agent's cost of information is determined by a function $C(\chi)$, which is increasing in $\chi$. For each $\chi$, we construct a corresponding $m \times n$ matrix $X$ of zeros and ones. The number of rows $m$ is the number of ones in $\chi$. If the $j^{\text {th }}$ entry of $\chi$ is 1 , then there is a row of $X$ that is all zeros, except for a one in the $j^{t h}$ position. When setting prices, the agent's information set $\mathcal{I}^{i}$ consists of his information choice $\chi^{i}$ and the vector of signals he observes $X \mathbf{z}^{i}$.

Equilibrium A Bayesian Nash Equilibrium is defined as a probability distribution $\mu$ over the feasible set of information choices $\Upsilon$, and a decision rule $p\left(\mathcal{I}^{i}\right)$, such that: $(i)$ given the distribution $\mu$, the decision rule $p\left(\mathcal{I}^{i}\right)$ solves $p\left(\mathcal{I}^{i}\right)=(1-r) E^{i}(s)+r E^{i}(\bar{p})$, and $(i i)$ the distribution $\mu$ assigns positive measure only to $\arg \max _{\chi \in \Upsilon}\left(E_{0}\left[u\left(p\left(\mathcal{I}^{i}\right), \bar{p}, s\right)\right]-C(\chi)\right)$.

Agents update their beliefs about the state, using these observed signals, according to Bayes' Law. $E_{0}(\cdot)$ denotes agents' expectations prior to observing any signals, while $E^{i}(\cdot)$ denotes an agent's posterior expectations, conditional on $\mathcal{I}^{i}$.

We focus on symmetric Nash equilibria, in which all agents follow the same (possibly mixed) strategy in stage one. Agents' information decisions will turn out to depend only on second moments of the distributions of $s$ and $\bar{p}$. Since these second moments are not affected by signal realizations, and are common knowledge in the model, information strategies will be common knowledge as well.

Ranking information choices When signals with different amounts of public and private information are available, it is not obvious which signal sets are more informative than others. Therefore, we define rankings of individual and aggregate information choices. The results use the following two definitions to describe how an agent's information choice changes when there is "more information acquisition".

Definition 1 For any $\chi, \chi^{\prime} \in \Upsilon, \chi$ is at least as informative as $\chi^{\prime}$, if and only if $\chi-\chi^{\prime} \geq 0$, entry-by-entry. 
Definition 2 For any $\mu, \mu^{\prime} \in \Delta(\Upsilon)$, the information choice distribution $\mu$ generates more aggregate information acquisition than $\mu^{\prime}$, if and only if $\mu$ first-order stochastically dominates $\mu^{\prime}$.

Thus, information choice $\chi$ is at least as informative as $\chi^{\prime}$, if every signal that an agent observes under $\chi^{\prime}$ is also observed under $\chi$. The second definition naturally extends this ranking of individual information choices to aggregate distributions over information choices.

\subsection{Results: the value of information}

Expectations and actions in stage 2 are conditioned on the agent's information sets. Agent $i$ 's information set includes the prior distribution of $s$ (common knowledge), the stage 1 information choice, and the realization of the acquired signals. We can solve the model by backwards induction: For any given distribution of information choices $\mu$, we characterize equilibrium strategies in stage 2 , and use this to determine expected payoffs in stage 1 . The results link the strategic motives in information acquisition to the strategic motives in actions.

\subsubsection{Bayesian Updating}

We begin by computing posterior beliefs, conditional on an information choice and signal realiza-

tions. We define a $k+1 \times 1$ vector of variables $\omega=\left[\begin{array}{cc}s & \mathbf{u}^{\prime}\end{array}\right]^{\prime}$. This is the relevant state variable because agents want to forecast the true state $s$ and the average action, which depends on common signal noise $\mathbf{u}$. The state $\omega$, and signals $X z^{i}$ are jointly normally distributed. Thus, conditional on observing $\mathcal{I}^{i}, \omega$ is normally distributed with posterior mean and variance-covariance matrix

$$
\begin{gathered}
E\left(\omega \mid \mathcal{I}^{i}\right)=\operatorname{Cov}\left(\omega, X \mathbf{z}^{i}\right)^{\prime} \operatorname{Var}\left(X \mathbf{z}^{i}\right)^{-1} X \mathbf{z}^{i} \\
\Sigma(\chi):=\operatorname{Var}\left(\omega \mid \mathcal{I}^{i}\right)=\operatorname{Var}(\omega)-\operatorname{Cov}\left(\omega, X \mathbf{z}^{i}\right)^{\prime} \operatorname{Var}\left(X \mathbf{z}^{i}\right)^{-1} \operatorname{Cov}\left(\omega, X \mathbf{z}^{i}\right) .
\end{gathered}
$$

This follows from standard formulas for the conditional distribution of normal variables. Appendix A derives these conditional moments and shows that $\operatorname{Var}(\omega)=I_{k+1}, \operatorname{Var}\left(X \mathbf{z}^{i}\right)=X \Gamma \Gamma^{\prime} X^{\prime}$, where $\Gamma=\left[\begin{array}{lll}\mathbf{1}_{n} & A & B\end{array}\right]$, and $\operatorname{Cov}\left(\omega, X \mathbf{z}^{i}\right)=\left[\mathbf{1}_{m}, X A\right]$.

For any probability distribution $\mu \in \Delta(\Upsilon)$ of the information choices made by all agents in the 
economy, we also define the average posterior variance-covariance matrix of $\omega: \bar{\Sigma}(\mu)=\int \Sigma(\chi) d \mu$, where integration is done entry-by-entry. This is a measure of the average level of uncertainty agents are facing.

The next lemma relates our ranking of information choices to the posterior variance-covariance matrices $\Sigma$ and $\bar{\Sigma}$. The proofs of this and all further results are in appendix A.

Lemma 1 ( $i)$ For any $\chi, \chi^{\prime} \in \Upsilon$, if information choice $\chi$ is more informative than $\chi^{\prime}$, then $\Sigma\left(\chi^{\prime}\right)-$ $\Sigma(\chi)$ is positive semi-definite.

(ii) For any $\mu, \mu^{\prime} \in \Delta(\Upsilon)$, if $\mu$ generates more aggregate information acquisition than $\mu^{\prime}$, then $\bar{\Sigma}\left(\mu^{\prime}\right)-\bar{\Sigma}(\mu)$ is positive semi-definite.

If $\chi$ is more informative than $\chi^{\prime}$, the posterior beliefs about $\omega$ conditioned on $\chi$ have lower variance than those conditioned on $\chi^{\prime}$. Since $\Sigma(\chi)$ corresponds to the mean squared forecast error, a more informative choice therefore leads to more accurate forecasts on average and to higher expected utility. Likewise, if a probability distribution $\mu \in \Delta(\Upsilon)$ of the information choices made by all agents leads to more aggregate information acquisition than an alternative distribution $\mu^{\prime}$, then the average posterior beliefs are on average more accurate under $\mu$, than under $\mu^{\prime}$. This result is useful because $\Sigma(\chi)$ and $\bar{\Sigma}(\mu)$ will show up in expected utility. The result tells us that these two terms are sufficient to capture the effects of individual and aggregate information acquisition.

The converse of lemma 1 only holds in special cases (for example, when all signals are independent of each other, but positively correlated across agents). In general it is possible for some information choice $\chi$ to generate a smaller variance-covariance matrix than $\chi^{\prime}$, even though some signals are observed under $\chi^{\prime}$ that are not observed under $\chi$.

\subsubsection{Optimal pricing strategies}

Solving by backwards induction means that we first solve the equilibrium in the price-setting game conditional on a given distribution of information choices, before solving the information choice problem. We conjecture that the following is the equilibrium price function and then verify that conjecture by showing that it satisfies the first-order condition. A separate technical appendix on 
the authors' websites shows that this equilibrium is unique. ${ }^{3}$

$$
p\left(\mathcal{I}^{i}\right)=(1-r) \mathbf{b}^{\prime} \sum_{k=0}^{\infty} r^{k} E^{i}\left(\bar{E}^{(k)}(\omega)\right)
$$

where $\mathbf{b}^{\prime}=\left[\begin{array}{lll}1 & 0 & \ldots\end{array}\right]$, and the average expectations operator is $\bar{E}^{(k)}(\cdot)=\bar{E} \bar{E}^{(k-1)}(\cdot)$, such that $\bar{E}^{(0)}(\omega)=\omega$, and $\bar{E}(\omega)=\int E^{i}(\omega) d i$. Averaging (2) across all $i$ and using the fact that the $\mathbf{v}^{i}$, s have mean zero reveals that the average expectation of $\omega$ is $\bar{E}(\omega)=[I-\bar{\Sigma}(\mu)] \omega$. Iterating on this equation tells us that $\bar{E}^{(k)}(\omega)=[I-\bar{\Sigma}(\mu)]^{k} \omega$. After substituting this expression into (4) and taking the limit of the infinite sum, the optimal pricing rule is

$$
p\left(\mathcal{I}^{i}\right)=(1-r) \mathbf{b}^{\prime}[(1-r) I+r \bar{\Sigma}(\mu)]^{-1} E^{i}(\omega)
$$

where $E^{i}(\omega)$ is given by (2). ${ }^{4}$ Averaging (5) and substituting in the formula for $\bar{E}(\omega)$ yields the average price $\bar{p}=(1-r) \mathbf{b}^{\prime}[(1-r) I+r \bar{\Sigma}(\mu)]^{-1}(I-\bar{\Sigma}(\mu)) \omega$. The resulting target price is $p^{*}=$ $(1-r) \mathbf{b}^{\prime}[(1-r) I+r \bar{\Sigma}(\mu)]^{-1} \omega$. Substituting $p\left(\mathcal{I}^{i}\right)$, and $p^{*}$ into (1) and taking an expectation delivers expected utility:

$$
E U(\chi, \mu) \equiv E^{i}\left(u\left(p_{i}, \bar{p}, s\right)\right)=-\mathbf{b}^{\prime}[(1-r) I+r \bar{\Sigma}(\mu)]^{-1} \Sigma(\chi)[(1-r) I+r \bar{\Sigma}(\mu)]^{-1} \mathbf{b}
$$

A linear function of more posterior variance $\Sigma$ decreases utility. In other words, more uncertainty makes agents less happy.

\subsubsection{Strategic incentives in information choice}

Our main result is that with decision complementarities, the value of information increases as there is more information acquisition; the opposite is true when decisions are strategic substitutes.

\footnotetext{
${ }^{3}$ See appendix A for derivations of the expressions that follow. The argument that (4) is the unique equilibrium originally appeared in Morris and Shin (2002). However, Dewan and Myatt (2007) and Angeletos and Pavan (2007a) pointed out gaps in their original proof, and Angeletos and Pavan (2007b) propose an alternative proof for uniqueness. The separate appendix simplifies and adapts their argument to our model.

${ }^{4}$ Strictly speaking, equation (4) is well-defined only if $r \in(-1,1)$. Equation (6), however remains well-defined for $r \leq-1$, and continues to constitute a solution to the agent's optimality condition, and hence an equilibrium.
} 
Proposition 1 Main Result: Suppose that $\chi$ is more informative than $\chi^{\prime}$, and $\mu$ leads to more information acquisition than $\mu^{\prime}$. Then

If there is no strategic interaction $(r=0)$, the value of additional information is independent of the other agents' information choices: $E U(\chi, \mu)-E U\left(\chi^{\prime}, \mu\right)=E U\left(\chi, \mu^{\prime}\right)-E U\left(\chi^{\prime}, \mu^{\prime}\right)$.

If decisions are complementary $(r>0)$, the value of additional information is increasing in the information acquisition of other agents: $E U(\chi, \mu)-E U\left(\chi^{\prime}, \mu\right)>E U\left(\chi, \mu^{\prime}\right)-E U\left(\chi^{\prime}, \mu^{\prime}\right)$.

If decisions are substitutes $(r<0)$, the value of additional information is decreasing in the information acquisition of other agents: $E U(\chi, \mu)-E U\left(\chi^{\prime}, \mu\right)<E U\left(\chi, \mu^{\prime}\right)-E U\left(\chi^{\prime}, \mu^{\prime}\right)$.

When $r>0$, acquiring information is a complement, regardless of whether it is public or private information. One might have imagined that complementarity only raises the value of public information. However, even when agents invest in private signals, more investment in information leads to a stronger covariance of prices with fundamentals, which in turn raises the value of information, if and only if decisions are complementary.

Thus the reason for the strategic motives in information acquisition comes from the covariance of the average prices with the state. As more agents become informed, their beliefs are closer to the truth and their prices are more reflective of the true state. For all $r<1$, the covariance of prices with the state is decreasing in $\bar{\Sigma}$ and thus increasing in the amount of aggregate information:

$$
\operatorname{cov}(\bar{p}, s \mid \chi)=(1-r) \mathbf{b}^{\prime}\left[(I-\bar{\Sigma}(\mu))^{-1}-r I\right]^{-1} \Sigma(\chi) \mathbf{b} .
$$

When prices are complements $(r \in(0,1))$, each firm's target price $p^{*}=(1-r) s+r \bar{p}$ puts positive weight on the state and the average price. When aggregate information $(\bar{\Sigma})$ increases, the higher covariance of prices and the state makes the variance of $p^{*}$ rise: $\operatorname{var}\left(p^{*}\right)=(1-r)^{2} \operatorname{var}(s)+$ $r^{2} \operatorname{var}(\bar{p})+2 r(1-r) \operatorname{cov}(\bar{p}, s)$. A more variable target price is a more uncertain target price. More uncertainty makes information more valuable. Conversely, when prices are strategic substitutes, the state and the average sign enter in the target price with opposite signs. Since $r(1-r)<0$, more covariance reduces the target price variance. This lowers the value of information. In summary, more aggregate information acquisition amplifies the agents' uncertainty about the target price when prices are complements and reduces uncertainty when prices are substitutes. This is why in 
the strategic motives in information acquisition mirror the strategic motives in price-setting.

This general theory of information acquisition underlies well-known results in other fields. For example, in Grossman and Stiglitz (1980), investment is a strategic substitute: Investors prefer purchasing assets that others don't want, because these assets have low prices. Similarly, actions are also substitutes in Cournot markets (Vives 1988) and winner-take-all forecasting contests (Ottaviani and Sorensen 2006). Each paper finds that information is a strategic substitute as well: the more information other agents purchase, the lower its value. Chamley (2006), Bullard, Evans, and Honkapohja (2007) and Froot, Scharfstein, and Stein (1992) construct a 'beauty contest' asset market, where investment is a strategic complement. They find that investors want to learn what other investors know. Our results explain why these results differ and how they can be extended to a broader class of settings: arbitrary combinations of public and private information, within a linear-quadratic model.

\subsection{Multiple Equilibria}

Adding information choice to games with strategic complementarity raises a new problem - multiple equilibria reappear in settings where the price-setting equilibrium would be unique. Two features of the information choice sets can produce multiple equilibria: discreteness and the choice over public or correlated information. This section shows that when either is present, multiple equilibria emerge.

\subsubsection{Discreteness in Information Choice}

Discreteness in information choice coupled with complementarity creates multiple equilibria. This result is not surprising because the same logic applies in games with discrete actions. We briefly present the result because it contrasts with the multiple equilibria generated by public information in the next section.

Agents decide whether to observe more or less information. Their choice set $\Upsilon$ contains $\chi$ and a less informative, less costly choice $\chi^{\prime}$. This is equivalent to endowing every agent with the signals corresponding to the positive entries of the vector $\chi^{\prime}$, and allowing them to purchase at a cost $C>0$ the additional signals corresponding to the positive entries of the vector $\chi-\chi^{\prime}$. 
If $r \in(0,1)$, there exist multiple equilibria. Let $\mu \in[0,1]$ denote the fraction of agents observing

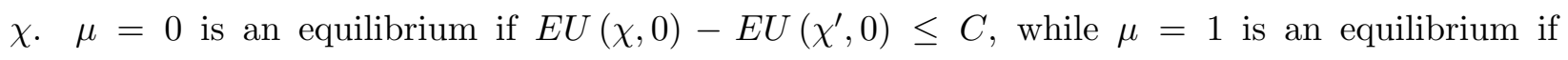
$E U(\chi, 1)-E U\left(\chi^{\prime}, 1\right) \geq C$. Proposition 1 says that this difference in marginal utilities is increasing in $\mu$ if and only if $r>0$. Thus, when $r>0$, there is a set of information $\operatorname{costs} C$ that satisfy both conditions and therefore generate multiple equilibria $(\mu=0, \mu=1$ and a symmetric mixed strategy equilibrium), only when prices are complements. If instead prices are substitutes, proposition 1 tells us that the value of additional information is monotonically decreasing in $\mu$. Therefore, for each $\operatorname{cost} C$ there exists either a pure strategy equilibrium ( $\mu=0$ or 1$)$ or a unique mixed strategy equilibrium $\mu \in(0,1)$ such that $E U(\chi, \mu)-E U\left(\chi^{\prime}, \mu\right)=C$.

\subsubsection{A model of near-continuous information choice}

To examine the effects of public or correlated signals, we consider the following special case of our model. Let $k=l=n, A=\gamma \delta^{-1 / 2} I_{n}$ and $B=\sqrt{1-\gamma^{2}} \delta^{-1 / 2} I_{n}$. This means that for a given agent, all signals are independent of each other; across agents, each signal contains a common noise component and a private noise component. The overall precision of any signal is $\delta$ and the correlation in the signal noise between any two agents is $\gamma^{2}$. We further suppose that the cost of acquiring any $m$ signals is $C(m \delta)$, where $C(\cdot)$ is differentiable, increasing and convex. We focus on the limiting case when $\delta \rightarrow 0$ and $n \rightarrow \infty$, so that the total number of signals becomes large, but the information content of any given signal vanishingly small.

This formulation embeds as special cases the case where all signals are potentially common $(\gamma=1)$, when signals are purely private $(\gamma=0)$ and any intermediate correlation.

Proposition 2 When information is near-continuous $(\delta \rightarrow 0)$, an information choice $\chi$ is sustainable as a symmetric pure-strategy equilibrium, if and only if the total signal precision $\phi=\delta \chi^{\prime} \chi$ satisfies

$$
\frac{1}{\left(1-r \gamma^{2}+(1-r) \phi\right)^{2}} \geq C^{\prime}(\phi) \geq \frac{\left(1-r \gamma^{2}\right)^{2}}{\left(1-r \gamma^{2}+(1-r) \phi\right)^{2}} .
$$

On the left side of the inequality is the marginal value of the last signal acquired, which is observed by all other agents. On the rights side is the marginal value of the next signal, which is not observed by others. If the marginal value of information is continuous, the left and right sides 
of the inequality are equal. This means that there is only one equilibrium $C^{\prime}(\phi)$ and thus a unique equilibrium level of signal precision. If the marginal value of information is discontinuous and there is an interval where the marginal value of private information is below the marginal cost, but the marginal value of public information is above it, multiple equilibria arise: It is optimal to acquire another bit of information if others do so as well.

Multiple equilibria arise in two ways: in the choice of which signals to observe and in the precision of the information acquired. The proposition bounds signal precision only. But many combinations of signals deliver the same precision. When information is private, every set of signals generates identical covariances with all aggregate variables, so this multiplicity is inconsequential. But when information is public or correlated, agents may decide to learn about variable $a$, in which case $p$ will be correlated with $a$. Or they could learn about $b$, in which case $p$ will be correlated with $b$. If $a$ and $b$ are macro aggregates, the information choice will determine their covariance with average actions. Multiple equilibria in signal precision is the topic we turn to next.

Multiple public/correlated information equilibria In price-setting games without information choice, endowing agents with heterogeneous information and eliminating discreteness in their choice sets is enough to guarantee unique outcomes. But when agents can choose to observe information that is potentially public or observe signals with correlated signal noise, multiple equilibria re-emerge. The existence of multiple equilibria follows directly from proposition 2 . When information is public or correlated $(\gamma \in(0,1])$, the left and right sides of the inequality differ by the factor $1 /\left(1-r \gamma^{2}\right)^{2}$. When $r>0,1>\left(1-r \gamma^{2}\right)^{2}$ and the left side is greater than the right. This means that there is a set of signal precisions $\phi$, whose cost is in the interval between the left and right side values, each of which is sustainable as an equilibrium.

One example of potentially public information is a newspaper. Imagine that each agent gets the same newspaper and chooses which pages of that paper to read. An additional page of the newspaper is only public information if other agents choose to read it. When $r>0$, public and private information have different marginal values; the marginal value of an additional page is discretely higher when that page is read by all other agents. This discontinuity creates multiple equilibria. 
Furthermore, unlike in Morris and Shin (1998), adding some information heterogeneity to agents' prior beliefs or to their signals will not unravel multiple equilibria. If firms observe signals with (imperfectly) correlated errors, then when they set the wrong prices, those prices will at least be similar and firms will benefit from being coordinated. Therefore, the marginal value of signals that are correlated with the ones others observe is strictly higher than the marginal value of additional information. The lower the correlation, the smaller the drop in the marginal value of extra signals that others do not observe, and the smaller the set of multiple equilibria (see figure 1). As a practical matter, a small degree of correlation is not very problematic because all of the information choices in the equilibrium set have almost identical precisions. The Morris and Shin (1998) refinement does not apply because signal heterogeneity affects beliefs about the level of $s$ and $\bar{p}$. But information demand depends only on the variance and covariance of $s$ and $\bar{p}$, which are common knowledge.

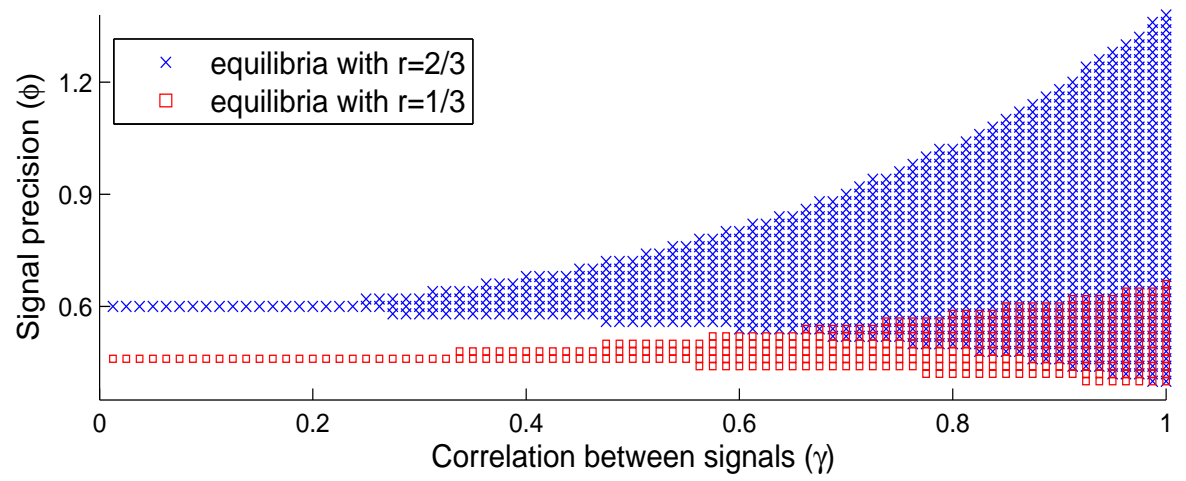

Figure 1: Choosing correlated information produces multiple equilibria. As the degree of correlation falls, the equilibrium set shrinks.

When prices are substitutes $r<0$, there is no symmetric pure strategy equilibrium in information acquisition: When $r<0$, no $\phi_{z}$ will satisfy the proposition's inequality. The discontinuity in the marginal value of information still generates a non-convexity. This non-convexity gives agents an incentive to invest in bits of information that other agents have not invested in. This is the same force that causes identical investors to acquire different information and thus hold different assets in Van Nieuwerburgh and Veldkamp (2007). The result also explains why information sharing is never optimal among Cournot competitors whose goods are substitutes, but is optimal for Bertrand competitors whose prices are complements (Vives 1984, 1988). 
Equilibrium Uniqueness: Choosing Private Signals Even in models with complementarity and information choice, multiple equilibria can be avoided. When agents choose the precision of private information, the equilibrium is unique. To see this result for symmetric pure-strategy equilibria, note that if $\gamma=0$ in proposition 2 , then the left and right sides of the inequality are equal. This pins down a unique value for the marginal cost of information $C^{\prime}(\phi)$. Since the cost function is assumed to be convex, there is a one-to-one mapping between marginal costs and number of signals. While proposition 2 suggests uniqueness of a pure strategy equilibrium, appendix A.5 generalizes this result by ruling out mixed or asymmetric equilibria.

One example of this type of information is the research of market analysts. Image an analyst choosing how large a research department to form. One research department's findings are uncorrelated with other departments' because researchers each discover independent signals about the truth. But with more researchers, the department's information will be more precise. Regardless of whether prices are complements or substitutes, when agents choose the precision of such a private signal from a continuous interval of potential precisions, there is a unique equilibrium.
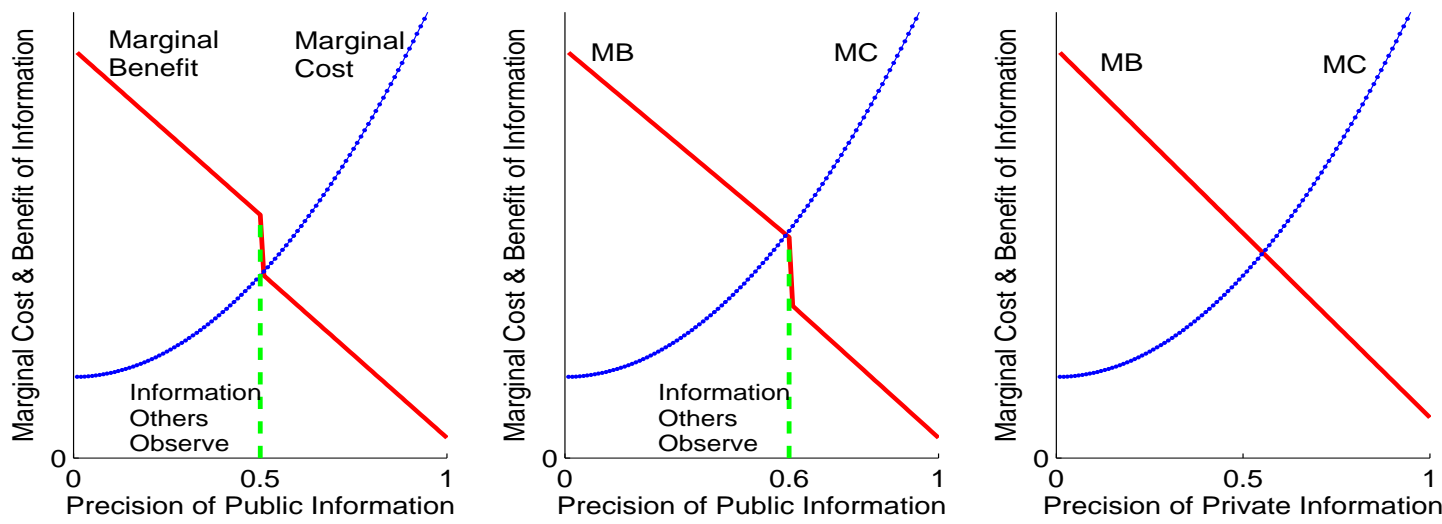

Figure 2: Public information choice (panels 1-2) produces multiple equilibria. The private information equilibrium (panel 3) is unique. In all three cases, prices are complements $(r>0)$. But the price-setting game alone would have a unique equilibrium.

Figure 2 illustrates how the private information choice model, where the marginal value of information is continuous, differs from the public information choice model where the marginal value of information falls discretely once an agent has observed more information than others have. Taken together, these results tell us that multiplicity is a robust phenomenon when agents 
play a coordination game with information choice. Simply adding private noise will not overturn the results. While restricting agents to choose information from menus of private signals with independent errors does guarantee uniqueness, it is a strong restriction.

\section{Dynamic Information Choice in Price-Setting Models}

While section 1 was about establishing our main results and showing why they were true, this section argues that our results are of practical relevance in price-setting models. Complementarity in price-setting creates complementarity in information acquisition, which helps create price rigidity. But the combination of complementarity and potentially public information also creates multiple equilibria. While our previous results tell us that uniqueness can be restored with private signals, section 2.4 details two ways this solution can be incorporated in models of price-setting.

Our results contribute to a long-standing debate in the dynamic price-setting literature (Ball and Romer 1989): Should firms who adjust prices infrequently synchronize or stagger their pricesetting? Only staggered price setting delivers the gradual response of aggregate price to monetary shocks observed in the data. Yet, the coordination motive in price-setting that many models employ to slow price adjustment also creates incentives to synchronize price-setting. A new generation of models, starting with Mankiw and Reis (2002), use information acquisition to slow price adjustment but ignore the strategic nature of information choice. By considering strategic information choice, we show that the coordination motive re-emerges, and with it, synchronized information acquisition. Of course, there are mechanisms that can sustain staggered price setting equilibria (Bhaskar 2002). Costly information models could use the same mechanisms to ensure staggered planning. But information choice, by itself, does not avoid the strategic problems that arise in standard pricesetting models.

The model, based on Reis (2006), is a dynamic version of the section 1 model. It builds on standard New Keynesian models. In order to focus on information choices, we analyze a simplified approximation to this micro-founded framework. In the underlying model, there is a continuum of monopolistically competitive firms, each producing a single intermediate good. These intermediates are bought by a representative household that combines them into a single final consumption good, 
using a CES technology with constant returns to scale. Intermediate firms continuously adjust the prices for their intermediate goods, based on the information they have about the underlying monetary shocks. The one non-standard piece of the model is that firms decide whether to pay a fixed cost to acquire information ("to plan"). Intermediate goods trade at pre-announced prices and labor inputs adjust to satisfy the resulting demand. All other market prices adjust to clear markets. Pricing complementarities in the intermediate sector result either from decreasing returns, or from equilibrium effects on wages and interest rates.

In the online technical appendix, we approximate this underlying model. To do this, we solve for aggregate allocations and market-clearing prices and wages as a function of the posted intermediate prices and the exogenous nominal shocks. Then, we determine the intermediate firm's payoffs, given their own price $p_{i}$, the aggregate price $p$, and the state of aggregate demand $s$. The objective function we use below is a quadratic approximation of firms' payoffs. This objective turns out to be similar to the one derived by Ball and Romer (1990) for a model with costly price adjustment.

\subsection{The model}

Time is continuous and infinite. There is a measure 1 continuum of firms. Each firm $i$ 's objective is to minimize the loss function

$$
\mathbb{E}_{0}\left\{\int_{0}^{\infty} e^{-\rho t}\left(p_{i}(t)-p^{*}(t)\right)^{2} d t+C \int_{0}^{\infty} e^{-\rho t} d D_{i}(t)\right\}
$$

where $p_{i}(t)$ denotes firm $i$ 's $\left(\log\right.$-)price at instant $t, D_{i}(t)$ is the process determining the dates at which the firm acquires information (plans): $d D_{i}(t)=1$ if the firm chooses to plan at instant $t$ and $d D_{i}(t)=0$ otherwise; $p^{*}(t)$ is an unknown, stochastic target price process that a firm with full information would set; $\rho>0$ is the firms' discount rate, and $C>0$ is the cost of planning.

Following the New Keynesian models of monopolistic competition, the target price is

$$
p^{*}(t)=(1-r) s(t)+r p(t)
$$

where $s(t)$ is the log of nominal demand at date $t, s(t)-p(t)$ is the log of real demand, $p(t)=$ 
$\int_{0}^{1} p_{i}(t) d i$ is the average price, and $r<1$ is a coefficient indicating the degree of strategic complementarity or "real rigidity" in price setting. For simplicity, we assume that demand is exogenous: $s(t)=\sigma Z(t)$, where $\sigma>0$ and $Z(t)$ is a standard Brownian motion.

Information Choices If firm $i$ last planned at date $\hat{\tau}$, it enters date $t$ with an information set $I_{i, t}=I_{\hat{\tau}}=\left\{Z\left(t^{\prime}\right): t^{\prime} \leq \hat{\tau}\right\}$, i.e. the firm knows the path of $Z$ up to and including date $\hat{\tau}$. If this firm plans in the current date $\left(d D_{i}(t)=1\right)$, its new information set contains all demand realizations prior to the current date: $I_{i, t+d t}=I_{t}=\left\{Z\left(t^{\prime}\right): t^{\prime} \leq t\right\} .^{5}$ If the firm does not plan $\left(d D_{i}(t)=0\right)$, its information set remains unchanged: $I_{i, t+d t}=I_{i, t}=I_{\hat{\tau}}$; it does not observe any new information about the state, including endogenous variables like the aggregate price level. This information structure comes from Reis (2006). It keeps the model tractable because the firm's last planning date pins down its information set. The idea is that firms can always observe these prices, but using them to infer demand or to re-compute their own optimal price requires a planning cost.

At any date $t$, the economy is characterized by the cross-sectional distribution of the firms' last planning dates; $\Lambda_{t}(\hat{\tau}) \in[0,1]$ denotes the fraction of firms who last planned prior to date $\hat{\tau}$. Then $1-\Lambda_{t}(\hat{\tau})$ is the fraction of firms who know all the demand realizations up to date $\hat{\tau}$ : $\left\{Z\left(t^{\prime}\right)\right\}_{t^{\prime} \leq \hat{\tau}}$. Let $d \Lambda_{t}(\hat{\tau})$ be the fraction (or density) of firms who last planned exactly on date $\hat{\tau}$ and let $D_{t}(\hat{\tau}) \in[0,1]$ be the probability that a firm who last planned at date $\hat{\tau}$ will plan in period $t$.

The evolution of $\Lambda_{t}(\hat{\tau})$ can be characterized recursively. The fraction of firms who will have planned since $\hat{\tau}$ at $t+d t$ is the fraction who planned between $\tau$ and $t$, plus the firms that last planned at any date before $\hat{\tau}$ and now choose to plan at date $t: \Lambda_{t+d t}(\hat{\tau})=\Lambda_{t}(\hat{\tau})-\int_{-\infty}^{\hat{\tau}} D_{t}(\tau) d \Lambda_{t}(\tau)$, for $\hat{\tau} \leq t$, with $\Lambda_{t}(t)=1$.

Equilibrium An equilibrium is a process of prices and planning choices by every firm $i,\left\{p_{i}(t), D_{i}(t)\right\}$, that are $I_{i, t}$-measurable and maximize (8), taking as given the choices of all other firms.

\footnotetext{
${ }^{5}$ This assumption implies that information acquired at date $t$ can not be used simultaneously for pricing decisions, or equivalently, at each instant, prices are set before planning choices are made.
} 
Prices and Indirect Utility Next, we characterize average price and target price processes, and expected losses, for arbitrary planning choices. At date $t$, a firm that last planned at date $\hat{\tau}$ sets its price equal to its expected target price at time $t: p_{i}(t)=E\left(p^{*}(t) \mid I_{\hat{\tau}}\right)=(1-r) E\left(s(t) \mid I_{\hat{\tau}}\right)+$ $r E\left(p(t) \mid I_{\hat{\tau}}\right)$. The average price is the average of the firms' expected target prices: $p(t)=$ $\int_{-\infty}^{t} E\left(p^{*}(t) \mid I_{\hat{\tau}}\right) d \Lambda_{t}(\hat{\tau})$. Lemma 1 expresses this as a function of the demand shocks $Z$.

Lemma 2 In equilibrium, $p^{*}(t)$ and $p(t)$ are characterized by

$$
p^{*}(t)=\sigma \int_{-\infty}^{t} \frac{1-r}{1-r+r \Lambda_{t}(\tau)} d Z(\tau) \quad \text { and } \quad p(t)=\sigma \int_{-\infty}^{t} \frac{\left(1-\Lambda_{t}(\tau)\right)(1-r)}{1-r+r \Lambda_{t}(\tau)} d Z(\tau)
$$

Since the expected value of unobserved demand innovations is 0 , the expected target price is the target price integrated over all the known demand realizations. For an agents whose known demand realizations end at date $\hat{\tau}$, this price is $E\left(p^{*}(t) \mid I_{\hat{\tau}}\right)=\sigma \int_{-\infty}^{\hat{\tau}}(1-r) /\left(1-r+r \Lambda_{t}(\tau)\right) d Z(\tau)$. It is the price firm $i$ chooses, $p_{i}(t)$.

From equation (9), we know that date- $t$ instantaneous expected loss is $E\left(p_{i}(t)-p^{*}(t) \mid I_{\hat{\tau}}\right)^{2}$. The difference between $p_{i}(t)$ and $p^{*}(t)$ depends on the demand shocks realized in between the date $\hat{\tau}$ when the firm last planned and $t: p_{i}(t)-p^{*}(t)=\sigma \int_{\hat{\tau}}^{t}(1-r) /\left(1-r+r \Lambda_{t}(\tau)\right) d Z(\tau)$. Since the variance of $Z(\tau)$ is 1 per unit time, the variance of the difference in prices is the coefficient squared:

$$
L(t, \hat{\tau})=\sigma^{2} \int_{\hat{\tau}}^{t}\left(\frac{1-r}{1-r+r \Lambda_{t}(\tau)}\right)^{2} d \tau
$$

We can use the instantaneous expected loss to construct a Bellman equation that characterizes the lifetime expected loss of a firm that plans in the current period $\hat{\tau}$ and optimally chooses its next planning date $\hat{\tau}^{\prime}$ :

$$
\mathcal{L}(\hat{\tau})=\min _{\hat{\tau}^{\prime} \geq \hat{\tau}}\left\{\int_{\hat{\tau}}^{\hat{\tau}^{\prime}} e^{-\rho(t-\hat{\tau})} L(t, \hat{\tau}) d t+e^{-\rho\left(\hat{\tau}^{\prime}-\hat{\tau}\right)}\left[C+\mathcal{L}\left(\hat{\tau}^{\prime}\right)\right]\right\} .
$$

The longer it has been since a firm has last planned, the stronger are its incentives to plan at the current date. An equilibrium is thus characterized by a threshold date $\tau^{*}(t)$, such that firms who have not planned since date $\tau^{*}(t)$ find it strictly optimal to plan at date $t$, while firms that have 
find not planning strictly optimal.

\subsection{Complementarity in Information Choice}

When prices are complements, $(r>0)$, there is a complementarity in planning. When prices are strategic substitutes $(r<0)$, the converse is true. This general principle in static models (proposition 1) re-appears in dynamic price-setting. Planning complementarity is important because it generates delays in price adjustment, which price-setting models are designed to explain.

To see where the planning complementarity arises, consider firms' per-period loss from not planning (10). The more firms are aware of the demand innovations that have occurred since the firm last planned, the higher is the per-period loss of not being aware of these innovations: $\partial L(t, \hat{\tau}) / \partial\left(1-\Lambda_{t}(\tau)\right)>0$; this holds for all planning dates $\tau \in(\hat{\tau}, t]$, if and only if prices are complementary $(r>0)$. This is the complementarity in planning decisions: the more recently other firms have planned, the higher is the cost to a firm of not planning in the current period.

To illustrate the effects of planning complementarity on price-setting, we next consider one equilibrium of this planning game, which has been the focus of previous work (Reis 2006). In this equilibrium, planning decisions are staggered, meaning that all firms plan after a fixed duration $T$, and over each interval of length $d t$, a fraction $T^{-1} d t$ of firms plan. This means that if $\tau<t-T$, then $\Lambda_{t}(\tau)=0$, but if $t-T<\tau \leq t, \Lambda_{t}(\tau)=1-(t-\tau) / T$. Therefore, a firm who last planned at date $\hat{\tau}$ has an instantaneous expect loss

$$
L(t, \hat{\tau})=\left\{\begin{array}{c}
\sigma^{2} \int_{\hat{\tau}}^{t}\left(\frac{1-r}{1-r(t-\tau) / T}\right)^{2} d \tau \text { if } \hat{\tau}>t-T \\
\sigma^{2} \int_{t-T}^{t}\left(\frac{1-r}{1-r(t-\tau) / T}\right)^{2} d \tau+\sigma^{2}(t-T-\hat{\tau}) \text { if } \hat{\tau}<t-T
\end{array}\right.
$$

Using (12), we can solve the firm's Bellman equation to determine when it is optimal to plan and characterize the equilibrium planning horizon $T$.

Proposition 3 A Staggered Planning Equilibrium: There is a unique staggered planning equilibrium. The equilibrium planning horizon $T$ is defined by $C=\sigma^{2}(1-r) T^{2} \int_{0}^{1} e^{-\rho T \theta} \frac{1-\theta}{1-r \theta} d \theta$. More complementarity (higher $r$ ) lengthens the equilibrium planning horizon $T$.

The pricing complementarity generates delays in price adjustment through two channels: First, 
because many other firms have prices based on old information, firms that do plan temper their reactions to recent information. This is the standard effect of complementarities in pricing models. Second, longer planning horizons means less information acquisition and less frequent price adjustment. Because many firms have old information, having outdated information is less costly, and frequent planning is less beneficial. This is planning complementarity. It amplifies the pricing complementarity to create long delays in price adjustment.

Reis (2006) uses this effect to match the empirical extent of price rigidity. Our theory results explain why it arises. As in the theory results, complementarity and covariance are mutually reinforcing effects. With more incomplete information, the covariance of average prices and demand falls. As that covariance falls, the incentive to plan for any individual firm falls because the aggregate price level becomes more predictable on the basis of their old information. This covariance effect is both the source of the information complementary and the key result of the price-setting model that allows it to match the data.

\subsection{Multiple Equilibria}

Although complementarity in price-setting and in information acquisition helps to slow price adjustment, it has a downside. The complementarity also generates multiple synchronized equilibria, in which all firms choose to plan at the same dates. The reason these equilibria arise is that when all firms plan at the same date, this introduces a discontinuity in the marginal value of planning at that date.

Without ruling out any other possibilities, we consider synchronized planning equilibria, in which all firms decide to plan at intervals of length $T$, and update their information at dates $\{T, 2 T, 3 T, \ldots\}$, for some $T>0$. Let $t$ denote the current date, and suppose all other firms last

planned at date $\tilde{\tau}\left(\Lambda_{t}(\tau)=0\right.$ if $\tau \leq \tilde{\tau}$ and $\Lambda_{t}(\tau)=1$ if $\left.\tau \in(\tilde{\tau}, t]\right)$. All firms therefore know the path of nominal spending $s(t)$ up until date $\tilde{\tau}$, while its more recent realizations remain unknown. For an individual firm that last planned in period $\hat{\tau}$, its period- $t$ expected loss is

$$
L(t, \hat{\tau})=\left\{\begin{array}{c}
\sigma^{2}(1-r)^{2}(t-\hat{\tau}) \text { if } \hat{\tau} \geq \tilde{\tau} \\
\sigma^{2}(1-r)^{2}(t-\tilde{\tau})+\sigma^{2}(\tilde{\tau}-\hat{\tau}) \text { if } \hat{\tau}<\tilde{\tau}
\end{array}\right.
$$


Consider now how $L(t, \hat{\tau})$ changes when all the others plan in the current period $(\tilde{\tau}$ shifts to $\tilde{\tau}=t$ ). If $r>0$, this leads to a discrete increase in $L(t, \hat{\tau})$, meaning that the loss from having outdated information rises discretely once the other firms have more recent information. This discrete jump generates multiple equilibria. When instead $r<0$, an agent's instantaneous loss decreases discretely when all the other firms plan; this in turn implies that synchronized planning cannot be sustained as an equilibrium, when pricing decisions are substitutes.

\section{Proposition 4 Multiple synchronized planning equilibria}

i. If $r \in(0,1)$, then any common planning horizon $T \in[\underline{T}, \bar{T}]$ is a synchronized planning equilibrium, where $\underline{T}$ solves $C=\sigma^{2} \underline{T}^{2} \int_{0}^{1} e^{-\rho \underline{T} \theta}(1-\theta) d \theta$ and $\bar{T}$ solves $C=(1-r)^{2} \sigma^{2} \bar{T}^{2} \int_{0}^{1} e^{-\rho \bar{T} \theta}(1-\theta) d \theta$.

ii. If $r=0$, there exists a unique synchronized planning equilibrium with a planning horizon $T^{*}$ implicitly defined by $C=\sigma^{2}\left(T^{*}\right)^{2} \int_{0}^{1} e^{-\rho T^{*} \theta}(1-\theta) d \theta$.

iii. If $r<0$, there does not exist a synchronized planning equilibrium.

The range of synchronized planning equilibria is bounded by two values: $\bar{T}$ is the planning horizon that would be optimal for any given firm, if all other firms were to never do any planning, while $\underline{T}$ is the planning horizon that would be optimal for a given firm, if all other firms always had complete information.

When actions are complements $(r>0)$, proposition 4 leaves open the possibility that there are

other synchronized planning equilibria with horizons outside the interval $[\underline{T}, \bar{T}]$. The actual range of synchronized planning equilibria can easily be computed numerically. For any $T>0$, define the last planning date $\hat{\tau} \in[0, T]$ as the firm's state variable, and let $\mathcal{L}(\hat{\tau})$ denote the lifetime expected loss of a firm that just planned at date $\hat{\tau}$.

The firm's optimal planning strategy is then characterized by solving the Bellman equation (11), where $\mathcal{L}(\hat{\tau})=\mathcal{L}(\hat{\tau}-T)$ for any $\hat{\tau} \geq T$, and $L(t, \hat{\tau})$ is given as above by (14), with $\tilde{\tau}=n T$, for $t \in(n T,(n+1) T]$. There exists a synchronized planning equilibrium if and only if the solution 
to this Bellman equation satisfies

$$
\mathcal{L}(0)=\frac{1}{1-e^{-\rho T}}\left\{(1-r)^{2} \sigma^{2} \int_{0}^{T} e^{-\rho t} t d t+e^{-\rho T} C\right\}
$$

Condition (14) implies that the proposed strategies do attain the optimum payoffs and hence constitute an equilibrium.

Appendix A.9 derives bounds $\underline{\underline{T}}$ and $\overline{\bar{T}}$ such that synchronized planning cannot be an equilibrium, whenever $T \notin[\underline{\underline{T}}, \overline{\bar{T}}]$. These bounds are constructed by considering two types of deviations that must be unprofitable in equilibrium: (1) marginal deviations that postpone or advance planning by a short time period and (2) deviations that skip a planning date or add extra dates, given

that all other firms plan at dates $\{T, 2 T, \ldots\}$. Numerical simulation confirms that any $T \in[\underline{\underline{T}}, \overline{\bar{T}}]$ is sustainable as a synchronized planning equilibrium. In other words, our necessary condition turns out to be sufficient as well.

How much does this matter for pricing? Multiple equilibria are not just a theoretical concern. They open up a range of predictions for planning horizons and price rigidity, for commonly used parameters. Figure 3 illustrates the set of equilibrium planning horizons sustainable for a range of complementarity parameters. If price complementarity $(r)$ is 0.85 , a level commonly used in new Keynesian models, possible planning horizons range from 3.2 to 64 quarters. The rate of price-adjustment varies greatly, depending on which of these equilibria firms select.

Multiple equilibria arise because the information firms observe when they plan is potentially public. Like reading an extra page of the newspaper, planning earlier than others plan delivers extra private information. When firms coordinate planning, they obtain public information, which is more valuable. The discontinuous value of information at the synchronized planning date produces multiplicity.

\subsection{Using Private Information To Make Equilibria Unique}

Staggered Planning with a Continuous Choice Space Two key features of Mankiw and Reis (2002) and Reis (2006) deliver a unique equilibrium and gradual price adjustment. First, 


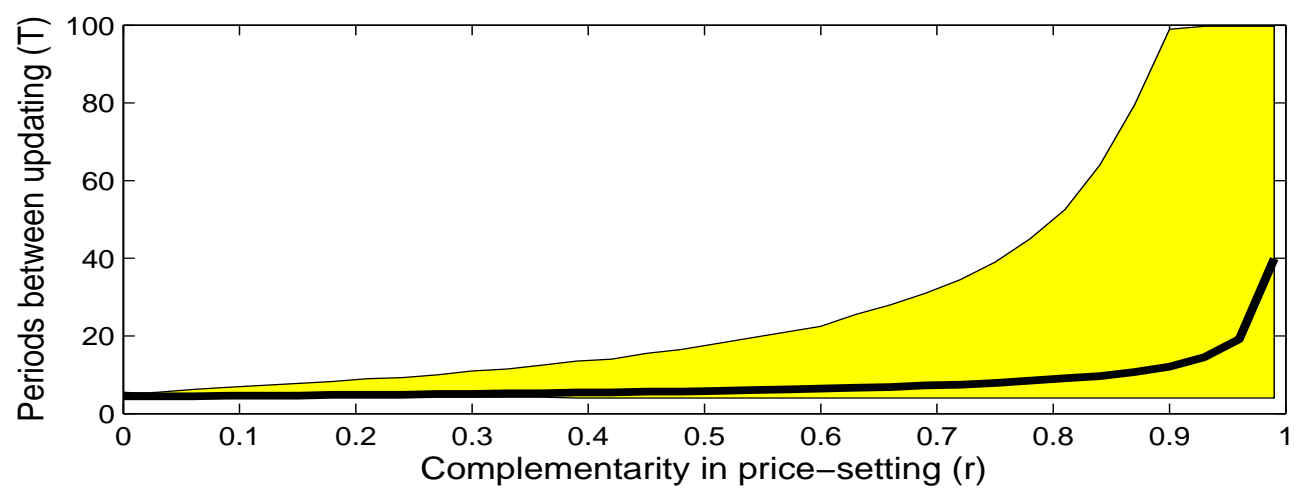

Figure 3: Equilibrium planning horizons for synchronized planning (shaded) and staggered planning (line). Two parameters being held fixed. One is the discount rate $\rho=0.01$, which implies a $4 \%$ annual rate of time-preference. Second is the ratio of the cost of information to the variance of demand shocks $C / \sigma^{2}=13$. This matches the 10-period staggered planning horizon in Reis (2006), for his calibrated degree of real rigidity, $r=0.85$.

they use continuous time. Second, they restrict the model to staggered planning equilibria where a constant fraction of firms plan at every date.

Since planning decisions are timing choices, the use of discrete time introduces discreteness in information choices. As in section 1.3.1, discreteness creates additional equilibria, both staggered and synchronized ones. ${ }^{6}$ In continuous time, multiple synchronized planning equilibria persist but the staggered equilibrium is unique. The reason is revealed in section 1.3.2. When firms synchronize their learning, they learn the same information as all other firms. Thus this is a public information choice problem which produces multiple equilibria. By staggering their planning, firms observe private information that others do not observe. The combination of private information and a continuous choice set delivers unique model predictions.

While restricting attention to staggered planning equilibria resolves the multiplicity problem, it does compromise the model's robustness. The predictions now depend crucially on the assumption that firms will stagger their planning. It is more desirable if the source of equilibrium uniqueness is micro-founded, supported by evidence, or is an integral part of the theory. The second example moves in this direction.

\footnotetext{
${ }^{6} \mathrm{~A}$ previous version of the paper examined the discrete-time version of this model.
} 
Rational Inattention An example of a price-setting model that has both private information and a continuous choice set is Mackowiak and Wiederholt (2006). They model rational agents who have limited information processing capability, as in Sims (2003), and who choose what shocks to observe more precisely and which to observe less precisely. The nature of the limited channel capacity is such that each agent observes signals with noise that is unique to them and is independent across agents. In other words, this way of modeling imperfect information ensures that signals are private and conditionally independent. While the mechanics of this model are quite similar to Reis (2006), Mackowiak and Wiederholt (2006) show that their equilibrium is unique. The uniqueness comes from the continuous nature of the precision allocation choice coupled with the private nature of the signal errors inherent to rational inattention.

\section{Conclusions}

Our results offer simple guidance on information choice in strategic settings. When actions are strategic substitutes, information is a substitute; when actions are complementary, information is a strategic complement. This strategic nature of information choice is central to price-setting models with costly information. Planning complementarity delays the adjustment of prices to shocks. But the same complementarity can lead firms to synchronize learning and adjust prices simultaneously. It can also generate multiple equilibria. These results do not make costly planning an untenable model. They do tell us what model features make the equilibrium unique: The information choice must be continuous and the signals must be private. This prescription is similar but not identical to that of Morris and Shin (1998). While a small amount of heterogeneity in beliefs produces unique outcomes in action games, information choice imposes the more stringent requirement that the signals in the information choice set are private, with independent errors.

Allowing agents to freely observe aggregate variables, such as prices, may or may not change the strategic incentives to acquire information. For example, in Grossman and Stiglitz (1980), actions are strategic substitutes. Prices introduce an additional free-rider effect, that reinforces substitutability in information choice: When others observe information, prices reveal some of it publicly, which reduces the value of additional information. In contrast, Barlevy and Veronesi 
(2000) generate information complementarity in the same setting, because more private information makes their asset prices less informative. Whether our conclusions are reversed or strengthened depends on the distribution of the noise in the asset's price. We leave the analysis of information complementarity, in the presence of an observable price, for future work.

All our results can be traced back to one root cause: Information acquisition changes the covariance of the state with the aggregate action. The same idea could explain changing covariances in macroeconomic variables. In fact, instability in the covariance of fundamentals and outcomes is a pervasive feature of macro time series, the most unstable variable being inflation (Stock and Watson 1996). More specifically, Olivei and Tenreyro (2007) document that monetary policy has stronger and faster effects in the beginning of the year and argue that this is because of synchronization in wage adjustments. A model of this phenomenon might resemble Cooper and John (1988) with regime changes in information choice. But instead of high-action and low-action regimes typical of action coordination games, actions covary strongly with an underlying state when it is observed precisely, and do not covary much when information about the state is scarce. Information demand might fluctuate for at least three reasons, 1) synchronized planning creates discrete changes in information demand at planning dates, 2) switches between multiple information equilibria, or sunspots, or 3) changes in information costs. Information choice offers one theory for why relationships between fundamentals and choices might fluctuate over time. Therefore, improving our understanding and prediction of information choice could help economists to better understand and forecast the relationships between macroeconomic fundamentals and aggregate actions. 


\section{References}

Allen, F., S. Morris, and H. Shin, 2006, "Beauty Contests and Bubbles," Review of Financial Studies, 19, 719-752.

Angeletos, G.-M., and A. Pavan, 2007a, "Efficient Use of Information and Social Value of Information," Econometrica, 75(4), 1103-1142.

Angeletos, G.-M., and A. Pavan, 2007b, "Policy with Dispersed Information," Working Paper.

Angeletos, G.-M., and I. Werning, 2006, "Crises and Prices: Information Aggregation, Multiplicity and Volatility," American Economic Review, 96(5), 1720-1736.

Ball, L., and D. Romer, 1989, "The equilibrium and optimal timing of price changes," Review of Economic Studies, 56, 179-198.

Ball, L., and D. Romer, 1990, "Real rigidities and the non-neutrality of money," Review of Economic Studies, 57, 183-203.

Barlevy, G., and P. Veronesi, 2000, "Information Acquisition in Financial Markets," Review of Economic Studies, 67, 79-90.

Bhaskar, V., 2002, "On Endogenously Staggered Prices," Review of Economic Studies, 69, 97-116.

Bikhchandani, S., D. Hirshleifer, and I. Welch, 1992, "A Theory of Fads, Fashion, Custom, and Cultural Change as Information Cascades," Journal of Political Economy, 100, 992-1026.

Bullard, J., G. Evans, and S. Honkapohja, 2007, "A Model of Near-Rational Exuberance," Working Paper.

Caplin, A., and J. Leahy, 1994, "Business as Usual, Market Crashes, and Wisdom After the Fact," American Economic Review, 84(3), 548-565.

Chamley, C., 2006, "Complementarities in information acquisition with short-term trades," Working Paper.

Cooper, R., and A. John, 1988, "Coordinating Coordination Failures in Keynesian Models," Quarterly Journal of Economics, 103, 441-63.

Cornand, C., and F. Heinemann, 2007, "Optimal Degree of Public Information Dissemination," Economic Journal, forthcoming.

Dewan, T., and D. Myatt, 2007, "The Qualities of Leadership: Direction, Communication, and Obfuscation," Working Paper.

Edmond, C., 2005, "Information Manipulation, Coordination and Regime Change," NYU Working Paper.

Farrell, J., and P. Klemperer, 2007, "Coordination and Lock-In: Competition with Switching Costs and Network Effects," in Mark Armstrong, and Robert Porter (ed.), Handbook of Industrial Organization, Elsevier. 
Froot, K., D. Scharfstein, and J. Stein, 1992, "Herd on the Street: Informational Inefficiencies in a Market with Short-Term Speculation," Journal of Finance, 47(4), 1461-84.

Goldstein, I., and A. Pauzner, 2005, "Demand Deposit Contracts and the Probability of Bank Runs," Journal of Finance, 60(3), 1293-1328.

Grossman, S., and J. Stiglitz, 1980, "On the Impossibility of Informationally Efficient Markets," American Economic Review, 70 (3), 393-408.

Hellwig, C., 2006, "Heterogeneous Information and the Benefits of Transparency," Working Paper.

Hellwig, C., A. Mukherji, and A. Tsyvinski, 2005, "Self-Fulfilling Currency Crises: The Role of Interest Rates," American Economic Review, 96 (5), 1769-1787.

Lorenzoni, G., 2006, "A Theory of Demand Shocks," MIT Working Paper.

Lucas, R., 1972, "Expectations and the Neutrality of Money," Journal of Economic Theory, 4, $103-124$.

Mackowiak, B., and M. Wiederholt, 2006, "Optimal Sticky Prices Under Rational Inattention," Northwestern University Working Paper.

Mankiw, G., and R. Reis, 2002, "Sticky Information Versus Sticky Prices: A Proposal to Replace the New Keynesian Phillips Curve," Quarterly Journal of Economics, 117, 1295-1328.

Morris, S., and H. Shin, 1998, "Unique Equilibrium in a Model of Self-Fulfilling Currency Attacks," American Economic Review, 88(3), 587-97.

Morris, S., and H. Shin, 2002, "The Social Value of Public Information," American Economic Review, 92, 1521-1534.

Moscarini, G., 2004, "Limited Information Capacity As A Source of Inertia," Journal of Economic Dynamics and Control, 28(10), 2003-2035.

Olivei, G., and S. Tenreyro, 2007, "The Timing of Monetary Policy Shocks," American Economic Review, 97(3), 636-663.

Ottaviani, M., and P. Sorensen, 2006, "The Strategy of Professional Forecasting," Journal of Financial Economics, 81(2), 441-466.

Reis, R., 2006, "Inattentive Producers," Review of Economic Studies, 73(3), 793-821.

Sims, C., 2003, "Implications of Rational Inattention," Journal of Monetary Economics, 50(3), 665-690.

Stock, J., and M. Watson, 1996, "Evidence on Structural Instability in Macroeconomic Time Series Relationships," Journal of Business and Economic Statistics, 14, 11-30.

Stromberg, D., 2001, "Mass Media and Public Policy," European Economic Review, 45, 652-663.

Van Nieuwerburgh, S., and L. Veldkamp, 2007, "Information Immobility and the Home Bias Puzzle," Working Paper. 
Veronesi, P., 1999, "Stock Market Overreactions to Bad News in Good Times: A Rational Expectations Equilibrium Model," Review of Financial Studies, 12(5), 975-1007.

Vives, X., 1984, "Duopoly Information Equilibrium: Cournot and Bertrand," Journal of Economic Theory, 34(1), 71-94.

Vives, X., 1988, "Aggregation of Information in Large Cournot Markets," Econometrica, 56, 851876.

Woodford, M., 2002, "Imperfect Common Knowledge and the Effects of Monetary Policy," in P. Aghion, R. Frydman, J. Stiglitz, and M. Woodford (ed.), Knowledge, Information, and Expectations in Modern Macroeconomics: In Honor of Edmund S. Phelps, Princeton Univ. Press.

Zeira, J., 1999, "Informational Overshooting, Booms and Crashes," Journal of Monetary Economics, 43, 237-257. 


\section{A Proofs and Derivations}

The expressions used for Bayesian updating (section 1.2.1) and equations (4) - (8) in the main text involve lots of algebraic manipulation at each step. Here, we provide additional detail for the reader who wants to replicate these expressions, along with proofs of the lemmas and propositions.

\section{A.1 Bayesian updating}

Equations (2) and (3) in the main text are standard formulas for the linear projection of one normal variable on another. We therefore need to derive the relevant unconditional variance and covariance terms. From $\mathbf{z}^{i}=$ $\mathbf{1}_{n} \cdot s+A \mathbf{u}+B \mathbf{v}^{i}$, we have

$$
\left(\begin{array}{c}
s \\
\mathbf{u} \\
X \mathbf{z}^{i}
\end{array}\right)=\left(\begin{array}{ccc}
1 & 0 & 0 \\
0 & I_{k} & 0 \\
X \mathbf{1}_{n} & X A & X B
\end{array}\right)\left(\begin{array}{c}
s \\
\mathbf{u} \\
\mathbf{v}^{i}
\end{array}\right)
$$

Now, recall that if $x \sim \mathcal{N}(\mu, \Sigma)$, then $C x \sim \mathcal{N}\left(C \mu, C \Sigma C^{\prime}\right)$. Since $\left(s, \mathbf{u}, \mathbf{v}^{i}\right)$ is jointly normally distributed with mean zero and variance-covariance matrix being the identity, it follows that $\left(s, \mathbf{u}, X \mathbf{z}^{i}\right)$ is jointly normally distributed with mean zero and variance-covariance matrix equal to

$$
\left(\begin{array}{ccc}
1 & 0 & 0 \\
0 & I_{k} & 0 \\
X \mathbf{1}_{n} & X A & X B
\end{array}\right)\left(\begin{array}{ccc}
1 & 0 & \mathbf{1}_{n}^{\prime} X^{\prime} \\
0 & I_{k} & A^{\prime} X^{\prime} \\
0 & 0 & B^{\prime} X^{\prime}
\end{array}\right)=\left(\begin{array}{ccc}
1 & 0 & \mathbf{1}_{n}^{\prime} X^{\prime} \\
0 & I_{k} & A^{\prime} X^{\prime} \\
X \mathbf{1}_{n} & X A & X \Gamma \Gamma^{\prime} X^{\prime}
\end{array}\right)
$$

where $\Gamma \equiv\left[\mathbf{1}_{n}, A, B\right]$. Using the definition of $\omega=\left[s, \mathbf{u}^{\prime}\right]^{\prime}$, we can therefore read off the relevant variance and covariance terms: $\operatorname{Var}(\omega)=\operatorname{Var}\left(\left[s, \mathbf{u}^{\prime}\right]^{\prime}\right)=I_{k+1}, \operatorname{Var}\left(X \mathbf{z}^{i}\right)=X \Gamma \Gamma^{\prime} X^{\prime}$, and $\operatorname{Cov}\left(\omega, X \mathbf{z}^{i}\right)=\left[X \mathbf{1}_{n}, X A\right]=\left[\mathbf{1}_{m}, X A\right]$, where we use the fact that $X \mathbf{1}_{n}=\mathbf{1}_{m}$, because $X$ is an $m \times n$ matrix with a one in each row.

\section{A.2 Proof of Lemma 1: Information set rankings and posterior variance}

Since part $(i i)$ is an immediate consequence of part $(i)$, we focus just on $(i)$. Consider therefore two information choices $\chi$ and $\chi^{\prime}$, such that $\chi-\chi^{\prime} \geq 0$ entry by entry, and $\chi_{m}-\chi_{m}^{\prime}=1$, for some $m$ (if $\chi=\chi^{\prime}$, the result holds trivially). Let $X_{1}=X_{\chi^{\prime}}$ and $X_{2}=X_{\chi-\chi^{\prime}}$, and notice that $X_{\chi}=\left(\begin{array}{cc}X_{1}^{\prime} & X_{2}^{\prime}\end{array}\right)^{\prime}$, up to a permutation of rows (since our definition - arbitrarily - orders signals in $X_{\chi} \mathbf{z}^{i}$ according to the same order as in $\left.\mathbf{z}^{i}\right)$.

Substituting in the variances and covariances into (3) results in

$$
\Sigma(\chi)=I_{1+k}-\left[\begin{array}{c}
\mathbf{1}_{n}^{\prime} \\
F^{\prime}
\end{array}\right] X^{\prime}\left\{X \Gamma \Gamma^{\prime} X^{\prime}\right\}^{-1} X\left[\begin{array}{ll}
\mathbf{1}_{n} & F
\end{array}\right] .
$$

Thus, $\Sigma\left(\chi^{\prime}\right)-\Sigma(\chi)$ is positive semi-definite, if and only if $X_{\chi}^{\prime}\left\{X_{\chi} \Gamma \Gamma^{\prime} X_{\chi}^{\prime}\right\}^{-1} X_{\chi}-X_{\chi^{\prime}}^{\prime}\left\{X_{\chi^{\prime}} \Gamma \Gamma^{\prime} X_{\chi^{\prime}}^{\prime}\right\}^{-1} X_{\chi^{\prime}}$ is positive semi-definite. To compute these matrices, notice that

$$
X_{\chi} \Gamma \Gamma^{\prime} X_{\chi}^{\prime}=\left(\begin{array}{c}
X_{1} \\
X_{2}
\end{array}\right) \Gamma \Gamma^{\prime}\left(\begin{array}{cc}
X_{1}^{\prime} & X_{2}^{\prime}
\end{array}\right)=\left(\begin{array}{cc}
F & G \\
G^{\prime} & H
\end{array}\right)
$$

where $F=X_{1} \Gamma \Gamma^{\prime} X_{1}^{\prime}, G=X_{1} \Gamma \Gamma^{\prime} X_{2}^{\prime}$, and $H=X_{2} \Gamma \Gamma^{\prime} X_{2}^{\prime}$. Inverting this matrix, we have

$$
\left\{X_{\chi} \Gamma \Gamma^{\prime} X_{\chi}^{\prime}\right\}^{-1}=\left(\begin{array}{cc}
\hat{F} & \hat{G} \\
\hat{G}^{\prime} & \hat{H}
\end{array}\right)
$$

where $\hat{F}=\left(F-G H^{-1} G^{\prime}\right)^{-1}, \hat{G}=-\left(F-G H^{-1} G^{\prime}\right)^{-1} G H^{-1}=-F^{-1} G\left(H-G^{\prime} F^{-1} G\right)^{-1}$, and $\hat{H}=\left(H-G^{\prime} F^{-1} G\right)^{-1}$. It follows that

$$
\begin{aligned}
X_{\chi}^{\prime}\left\{X_{\chi} \Gamma \Gamma^{\prime} X_{\chi}^{\prime}\right\}^{-1} X_{\chi}= & \left(\begin{array}{cc}
X_{1}^{\prime} & X_{2}^{\prime}
\end{array}\right)\left(\begin{array}{cc}
\hat{F} & \hat{G} \\
\hat{G}^{\prime} & \hat{H}
\end{array}\right)\left(\begin{array}{c}
X_{1} \\
X_{2}
\end{array}\right)=X_{1}^{\prime} \hat{F} X_{1}+X_{2}^{\prime} \hat{G}^{\prime} X_{1}+X_{1}^{\prime} \hat{G} X_{2}+X_{2}^{\prime} \hat{H} X_{2} \\
= & X_{1}^{\prime} \hat{F} X_{1}-X_{2}^{\prime}\left(H-G^{\prime} F^{-1} G\right)^{-1} G^{\prime} F^{-1} X_{1}-X_{1}^{\prime} F^{-1} G\left(H-G^{\prime} F^{-1} G\right)^{-1} X_{2} \\
& +X_{2}^{\prime}\left(H-G^{\prime} F^{-1} G\right)^{-1} X_{2} \\
= & X_{1}^{\prime} \hat{F} X_{1}-X_{1}^{\prime} F^{-1} G\left(H-G^{\prime} F^{-1} G\right)^{-1} G^{\prime} F^{-1} X_{1}
\end{aligned}
$$




$$
\begin{aligned}
& +\left(X_{2}^{\prime}-X_{1}^{\prime} F^{-1} G\right)\left(H-G^{\prime} F^{-1} G\right)^{-1}\left(X_{2}-G^{\prime} F^{-1} X_{1}\right) \\
= & X_{1}^{\prime}\left[\left(F-G H^{-1} G^{\prime}\right)^{-1}-\left(F-G H^{-1} G^{\prime}\right)^{-1} G H^{-1} G^{\prime} F^{-1}\right] X_{1} \\
& +\left(X_{2}^{\prime}-X_{1}^{\prime} F^{-1} G\right)\left(H-G^{\prime} F^{-1} G\right)^{-1}\left(X_{2}-G^{\prime} F^{-1} X_{1}\right) \\
= & X_{1}^{\prime} F^{-1} X_{1}+\left(X_{2}^{\prime}-X_{1}^{\prime} F^{-1} G\right)\left(H-G^{\prime} F^{-1} G\right)^{-1}\left(X_{2}-G^{\prime} F^{-1} X_{1}\right) .
\end{aligned}
$$

On the other hand, $X_{\chi^{\prime}}^{\prime}\left\{X_{\chi^{\prime}} \Gamma \Gamma^{\prime} X_{\chi^{\prime}}^{\prime}\right\}^{-1} X_{\chi^{\prime}}=X_{1}^{\prime}\left\{X_{1} \Gamma \Gamma^{\prime} X_{1}^{\prime}\right\}^{-1} X_{1}=X_{1}^{\prime} F^{-1} X_{1}$. It therefore suffices to show that $\left(H-G^{\prime} F^{-1} G\right)^{-1}$, or equivalently $H-G^{\prime} F^{-1} G$, is positive semi-definite. Now,

$$
\begin{aligned}
H-G^{\prime} F^{-1} G & =X_{2} \Gamma \Gamma^{\prime} X_{2}^{\prime}-X_{2} \Gamma \Gamma^{\prime} X_{1}^{\prime}\left\{X_{1} \Gamma \Gamma^{\prime} X_{1}^{\prime}\right\}^{-1} X_{1} \Gamma \Gamma^{\prime} X_{2}^{\prime} \\
& =X_{2} \Gamma\left\{I-\Gamma^{\prime} X_{1}^{\prime}\left\{X_{1} \Gamma \Gamma^{\prime} X_{1}^{\prime}\right\}^{-1} X_{1} \Gamma\right\} \Gamma^{\prime} X_{2}^{\prime} .
\end{aligned}
$$

The matrix $I-\Gamma^{\prime} X_{1}^{\prime}\left\{X_{1} \Gamma \Gamma^{\prime} X_{1}^{\prime}\right\}^{-1} X_{1} \Gamma$ is symmetric and idempotent, and therefore it is positive semi-definite, and so is $H-G^{\prime} F^{-1} G$.

\section{A.3 Derivation of equations 4-7}

Derivation of equation 4 and proof that this characterizes an equilibrium Averaging the agents' first-order condition across all agents, we find that the average price must satisfy $\bar{p}=(1-r) \bar{E}(s)+r \bar{E}(\bar{p})$. If we repeatedly substitute this expression into the agents' first-order condition, we find that any equilibrium price must satisfy

$$
p\left(\mathcal{I}^{i}\right)=(1-r) E^{i}(s)+r(1-r) E^{i} \bar{E}(s)+r^{2} E^{i} \bar{E}(\bar{p})=(1-r) \sum_{k=0}^{K} r^{k} E^{i}\left(\bar{E}^{(k)}(s)\right)+r^{K+1} E^{i}\left(\bar{E}^{(K)}(\bar{p})\right)
$$

If we let $K$ go to infinity and conjecture that $\lim _{K \rightarrow \infty} r^{K+1} E^{i}\left(\bar{E}^{(K)}(\bar{p})\right)=0$, we arrive at the definition of $\hat{p}$ that is given in the main text. We can check that this indeed constitutes an equilibrium, by substituting it back into the right-hand-side of the agents' first-order condition: Averaging $\hat{p}$ across all $i$, we find $\bar{p}=(1-r) \sum_{k=0}^{\infty} r^{k}\left(\bar{E}^{(k+1)}(s)\right)$, so the right side of the agents' first-order condition becomes

$$
\begin{aligned}
(1-r) E^{i}(s)+r E^{i}(\bar{p}) & =(1-r) E^{i}(s)+r E^{i}\left((1-r) \sum_{k=0}^{\infty} r^{k} \bar{E}^{(k+1)}(s)\right. \\
& =(1-r) E^{i}(s)+(1-r) \sum_{k=0}^{\infty} r^{k+1} E^{i}\left(\bar{E}^{(k+1)}(s)\right) \\
& =(1-r) E^{i}(s)+(1-r) \sum_{k=1}^{\infty} r^{k} E^{i}\left(\bar{E}^{(k)}(s)\right) \\
& =(1-r) \sum_{k=0}^{\infty} r^{k} E^{i}\left(\bar{E}^{(k)}(s)\right)=\hat{p}\left(\mathcal{I}^{i}\right),
\end{aligned}
$$

where we have first pulled the expectation and $r$ inside the summation, then changed the index of the summation, and finally combined the terms.

Derive equation 5 Equation (2) in the main text gives us $E\left(\omega \mid X \mathbf{z}^{i}\right)=\left[\mathbf{1}_{m}, X A\right]^{\prime}\left[X \Gamma \Gamma^{\prime} X^{\prime}\right]^{-1} X \mathbf{z}^{i}$. Integrating this expression across all agents, we find the average expectations of $\omega$ :

$$
\begin{aligned}
\bar{E}(\omega) & =\int_{i} E\left(\omega \mid X \mathbf{z}^{i}\right) d \mu\left(\chi^{i}\right)=\iint\left[\mathbf{1}_{m}, X A\right]^{\prime}\left[X \Gamma \Gamma^{\prime} X^{\prime}\right]^{-1} X \mathbf{z}^{i} d \Phi\left(\mathbf{z}^{i} \mid \omega\right) d \mu(\chi) \\
& =\int\left[\mathbf{1}_{m}, X A\right]^{\prime}\left[X \Gamma \Gamma^{\prime} X^{\prime}\right]^{-1} X \int \mathbf{z}^{i} d \Phi\left(\mathbf{z}^{i} \mid \omega\right) d \mu(\chi) .
\end{aligned}
$$

Next we solve the inside integral. Since $\mathbf{z}^{i}=\left[\mathbf{1}_{n}, A\right] \omega+B \mathbf{v}^{i}$, we can rewrite this as

$$
\int \mathbf{z}^{i} d \Phi\left(\mathbf{z}^{i} \mid \omega\right)=\int\left[\left[\mathbf{1}_{n}, A\right] \omega+B \mathbf{v}^{i}\right] d \Phi\left(\mathbf{v}^{i}\right)=\left[\mathbf{1}_{n}, A\right] \omega+B \int \mathbf{v}^{i} d \Phi\left(\mathbf{v}^{i}\right)=\left[\mathbf{1}_{n}, A\right] \omega,
$$


due to the fact that the cross-sectional mean of the $\mathbf{v}^{i}$ 's is zero. We substitute this back into the expression for $\bar{E}(\omega)$ and solve the outer integral:

$$
\begin{aligned}
\bar{E}(\omega) & =\int\left[\mathbf{1}_{m}, X A\right]^{\prime}\left[X \Gamma \Gamma^{\prime} X^{\prime}\right]^{-1} X\left[\mathbf{1}_{n}, A\right] \omega d \mu(\chi)=\int\left[\mathbf{1}_{m}, X A\right]^{\prime}\left[X \Gamma \Gamma^{\prime} X^{\prime}\right]^{-1} X\left[\mathbf{1}_{n}, A\right] d \mu(\chi) \cdot \omega \\
& =\int\left[\mathbf{1}_{m}, X A\right]^{\prime}\left[X \Gamma \Gamma^{\prime} X^{\prime}\right]^{-1}\left[\mathbf{1}_{m}, X A\right] d \mu(\chi) \cdot \omega,
\end{aligned}
$$

where we have once again used the fact that $X \mathbf{1}_{n}=\mathbf{1}_{m}$. Now, using equation (3) from the main text, which states that $\Sigma\left(\chi^{i}\right)=I-\left[\mathbf{1}_{m}, X A\right]^{\prime}\left[X \Gamma \Gamma^{\prime} X^{\prime}\right]^{-1}\left[\mathbf{1}_{m}, X A\right]$, along with the definition $\bar{\Sigma}(\mu)=\int \Sigma\left(\chi^{i}\right) d \mu\left(\chi^{i}\right)$, we find

$$
\bar{E}(\omega)=\left[I-\int \Sigma\left(\chi^{i}\right) d \mu\left(\chi^{i}\right)\right] \cdot \omega=[I-\bar{\Sigma}(\mu)] \omega .
$$

We next prove by induction that $\bar{E}^{(k)}(\omega)=[I-\bar{\Sigma}(\mu)]^{k} \omega$. For this, notice that the result already holds for $k=0$ and $k=1$, and suppose that it also holds for arbitrary $k-1$. Then,

$$
\begin{aligned}
\bar{E}^{(k)}(\omega) & =\int_{i} E\left[\bar{E}^{(k-1)}(\omega) \mid X \mathbf{z}^{i}\right] d \mu\left(\chi^{i}\right)=\int_{i} E\left[[I-\bar{\Sigma}(\mu)]^{k-1} \omega \mid X \mathbf{z}^{i}\right] d \mu\left(\chi^{i}\right) \\
& =[I-\bar{\Sigma}(\mu)]^{k-1} \int_{i} E\left[\omega \mid X \mathbf{z}^{i}\right] d \mu\left(\chi^{i}\right)=[I-\bar{\Sigma}(\mu)]^{k-1}[I-\bar{\Sigma}(\mu)] \omega=[I-\bar{\Sigma}(\mu)]^{k} \omega .
\end{aligned}
$$

The first equality follows from the definition of $\bar{E}(\cdot)$, the second uses the induction hypothesis, the third takes the non-stochastic matrix $[I-\bar{\Sigma}(\mu)]^{k-1}$ outside the expectation, and the fourth makes use of our above characterization for $\bar{E}(\omega)$.

Finally, we solve out for the pricing function in equation (4). We first substitute $s=\mathbf{b}^{\prime} \omega$ into the equation and take $\mathbf{b}$ outside the expectations. We then substitute in for the sequence of higher-order expectations $\bar{E}^{(k)}(\omega)$, and solve out the infinite sum expression:

$$
\begin{aligned}
\hat{p}\left(\mathcal{I}^{i}\right) & =(1-r) \mathbf{b}^{\prime} \sum_{k=0}^{\infty} r^{k} E\left(\bar{E}^{(k)}(\omega) \mid \mathcal{I}^{i}\right)=(1-r) \mathbf{b}^{\prime} \sum_{k=0}^{\infty} r^{k} E\left([I-\bar{\Sigma}(\mu)]^{k} \omega \mid \mathcal{I}^{i}\right) \\
& =(1-r) \mathbf{b}^{\prime} \sum_{k=0}^{\infty} r^{k}[I-\bar{\Sigma}(\mu)]^{k} E\left(\omega \mid \mathcal{I}^{i}\right)=(1-r) \mathbf{b}^{\prime}[I-r[I-\bar{\Sigma}(\mu)]]^{-1} E\left(\omega \mid \mathcal{I}^{i}\right) .
\end{aligned}
$$

Derive $p^{*} \quad$ The target price $p^{*}$ is defined as $p^{*}=(1-r) s+r \bar{p}$. Substituting $s=\mathbf{b}^{\prime} \omega$ and $\bar{p}=(1-r) \mathbf{b}^{\prime}[(1-r) I+r \bar{\Sigma}(\mu)]^{-1}(I-\bar{\Sigma}(\mu)) \omega$ into this expression, we find

$$
\begin{aligned}
p^{*} & =(1-r) \mathbf{b}^{\prime} \omega+r(1-r) \mathbf{b}^{\prime}[(1-r) I+r \bar{\Sigma}(\mu)]^{-1}(I-\bar{\Sigma}(\mu)) \omega \\
& =(1-r) \mathbf{b}^{\prime}\left\{I+r[(1-r) I+r \bar{\Sigma}(\mu)]^{-1}(I-\bar{\Sigma}(\mu))\right\} \omega \\
& =(1-r) \mathbf{b}^{\prime}[(1-r) I+r \bar{\Sigma}(\mu)]^{-1}\{(1-r) I+r \bar{\Sigma}(\mu)+r(I-\bar{\Sigma}(\mu))\} \omega \\
& =(1-r) \mathbf{b}^{\prime}[(1-r) I+r \bar{\Sigma}(\mu)]^{-1} \omega
\end{aligned}
$$

Derive equation 6 Equation (1) defines realized utility. Taking its expectation, conditional on agent $i$ 's information,

$$
E^{i} U=E^{i}\left[-\frac{1}{(1-r)^{2}}\left(p^{*}-p_{i}\right)^{2}\right]=-\frac{1}{(1-r)^{2}} E^{i}\left\{\left(p^{*}-p_{i}\right)\left(p^{*}-p_{i}\right)^{\prime}\right\} .
$$

From the equilibrium characterization, we have $p^{*}-p_{i}=(1-r) \mathbf{b}^{\prime}[(1-r) I+r \bar{\Sigma}(\mu)]^{-1}\left(\omega-E^{i}(\omega)\right)$. We substitute this into $E^{i} U$, and make use of the fact that $E^{i}\left\{\left(\omega-E^{i}(\omega)\right)\left(\omega^{\prime}-E^{i}\left(\omega^{\prime}\right)\right)\right\}$ corresponds to the posterior variance covariance matrix, $\Sigma(\chi)$ :

$$
\begin{aligned}
E^{i} U & =-E^{i}\left\{\mathbf{b}^{\prime}[(1-r) I+r \bar{\Sigma}(\mu)]^{-1}\left(\omega-E^{i}(\omega)\right)\left(\omega^{\prime}-E^{i}\left(\omega^{\prime}\right)\right)[(1-r) I+r \bar{\Sigma}(\mu)]^{-1} \mathbf{b}\right\} \\
& =-\mathbf{b}^{\prime}[(1-r) I+r \bar{\Sigma}(\mu)]^{-1} E^{i}\left\{\left(\omega-E^{i}(\omega)\right)\left(\omega^{\prime}-E^{i}\left(\omega^{\prime}\right)\right)\right\}[(1-r) I+r \bar{\Sigma}(\mu)]^{-1} \mathbf{b} \\
& =-\mathbf{b}^{\prime}[(1-r) I+r \bar{\Sigma}(\mu)]^{-1} \Sigma(\chi)[(1-r) I+r \bar{\Sigma}(\mu)]^{-1} \mathbf{b}
\end{aligned}
$$

Since there is no ex ante uncertainty about these second moments, this is also agents' unconditional expected utility. 
Derive equation $7 \quad$ Using the fact that $s=\mathbf{b}^{\prime} \omega$ and the derivation of $\bar{p}, \operatorname{cov}(\bar{p}, s)$ becomes

$$
\operatorname{cov}\left(\bar{p}, \mathbf{b}^{\prime} \omega\right)=\operatorname{cov}\left((1-r) \mathbf{b}^{\prime}[(1-r) I+r \bar{\Sigma}(\mu)]^{-1}(I-\bar{\Sigma}(\mu)) \omega, \mathbf{b}^{\prime} \omega\right) .
$$

Pulling the various matrices out of this covariance expression, and once again using $\operatorname{cov}(\omega, \omega)=E\left\{\left(\omega-E^{i}(\omega)\right)\left(\omega^{\prime}-E^{i}\left(\omega^{\prime}\right)\right)\right\}=\Sigma(\chi)$, we find

$$
\begin{aligned}
\operatorname{cov}\left(\bar{p}, \mathbf{b}^{\prime} \omega\right) & =(1-r) \mathbf{b}^{\prime}[(1-r) I+r \bar{\Sigma}(\mu)]^{-1}(I-\bar{\Sigma}(\mu)) \operatorname{cov}(\omega ; \omega) \mathbf{b} \\
& =(1-r) \mathbf{b}^{\prime}[(1-r) I+r \bar{\Sigma}(\mu)]^{-1}(I-\bar{\Sigma}(\mu)) \Sigma(\chi) \mathbf{b} .
\end{aligned}
$$

The same expression with $I$ instead of $\Sigma(\chi)$ characterizes the unconditional covariance of $\bar{p}$ and $s$.

\section{A.4 Proof of Proposition 1: Strategic incentives in information choice}

The proof for no strategic interactions $(r=0)$ follows directly from the expected utility function.

For the case where $r \neq 0$, define $\Psi(\mu) \equiv I-\bar{\Sigma}(\mu)$. Recall that $\mathbf{b}^{\prime}=\left[\begin{array}{lll}1 & 0 \ldots 0\end{array}\right]$. Rearranging terms, we have

$$
\begin{aligned}
& E U(\chi, \mu)-E U\left(\chi^{\prime}, \mu\right)-\left(E U\left(\chi, \mu^{\prime}\right)-E U\left(\chi^{\prime}, \mu^{\prime}\right)\right) \\
= & \mathbf{b}^{\prime}[I-r \Psi(\mu)]^{-1}\left[\Sigma\left(\chi^{\prime}\right)-\Sigma(\chi)\right][I-r \Psi(\mu)]^{-1} \mathbf{b} \\
& -\mathbf{b}^{\prime}\left[I-r \Psi\left(\mu^{\prime}\right)\right]^{-1}\left[\Sigma\left(\chi^{\prime}\right)-\Sigma(\chi)\right]\left[I-r \Psi\left(\mu^{\prime}\right)\right]^{-1} \mathbf{b} \\
= & \mathbf{b}^{\prime}\left\{[I-r \Psi(\mu)]^{-1}-\left[I-r \Psi\left(\mu^{\prime}\right)\right]^{-1}\right\}\left[\Sigma\left(\chi^{\prime}\right)-\Sigma(\chi)\right][I-r \Psi(\mu)]^{-1} \mathbf{b} \\
& +\mathbf{b}^{\prime}\left[I-r \Psi\left(\mu^{\prime}\right)\right]^{-1}\left[\Sigma\left(\chi^{\prime}\right)-\Sigma(\chi)\right]\left\{[I-r \Psi(\mu)]^{-1}-\left[I-r \Psi\left(\mu^{\prime}\right)\right]^{-1}\right\} \mathbf{b} \\
= & \mathbf{b}^{\prime}\left\{[I-r \Psi(\mu)]^{-1}-\left[I-r \Psi\left(\mu^{\prime}\right)\right]^{-1}\right\}\left[\Sigma\left(\chi^{\prime}\right)-\Sigma(\chi)\right][I-r \Psi(\mu)]^{-1} \mathbf{b} \\
& +\mathbf{b}^{\prime}\left\{[I-r \Psi(\mu)]^{-1}-\left[I-r \Psi\left(\mu^{\prime}\right)\right]^{-1}\right\}\left[\Sigma\left(\chi^{\prime}\right)-\Sigma(\chi)\right]\left[I-r \Psi\left(\mu^{\prime}\right)\right]^{-1} \mathbf{b} \\
= & \mathbf{b}^{\prime}\left\{[I-r \Psi(\mu)]^{-1}-\left[I-r \Psi\left(\mu^{\prime}\right)\right]^{-1}\right\}\left[\Sigma\left(\chi^{\prime}\right)-\Sigma(\chi)\right]\left\{[I-r \Psi(\mu)]^{-1}+\left[I-r \Psi\left(\mu^{\prime}\right)\right]^{-1}\right\} \mathbf{b}
\end{aligned}
$$

where the second-to-last step follows from symmetry (of a $1 \times 1$ matrix). In what follows, we use $>0$ to signify that a matrix is positive definite and $<0$ to mean negative definite. We also use the fact that sums and products of positive definite matrices are positive definite. Clearly, $\Sigma\left(\chi^{\prime}\right)-\Sigma(\chi)>0$ and $[I-r \Psi(\mu)]^{-1}+\left[I-r \Psi\left(\mu^{\prime}\right)\right]^{-1}>0$. Moreover, if $r>0, \Psi(\mu)-\Psi\left(\mu^{\prime}\right)>0$ implies $r\left(\Psi(\mu)-\Psi\left(\mu^{\prime}\right)\right)>0$ and $[I-r \Psi(\mu)]^{-1}-\left[I-r \Psi\left(\mu^{\prime}\right)\right]^{-1}>0$. Since any positive-definite matrix, pre- and post-multiplied by the same vector yields a positive scalar, the overall expression is positive whenever $r>0$. Likewise, if $r<0,[I-r \Psi(\mu)]^{-1}-\left[I-r \Psi\left(\mu^{\prime}\right)\right]^{-1}<0$ implying that the overall expression is negative.

\section{A.5 Proof of Proposition 2: Multiple equilibria}

The definition of $A$ and $B$ implies that $\Gamma \Gamma^{\prime}=\mathbf{1}_{n} \mathbf{1}_{n}^{\prime}+\delta^{-1} I_{n}$, from which it follows that for any $\chi \in\{0,1\}^{n}$, $X_{\chi} \Gamma \Gamma^{\prime} X_{\chi}^{\prime}=\mathbf{1}_{\tilde{n}} \mathbf{1}_{\tilde{n}}^{\prime}+\delta^{-1} I_{\tilde{n}}$, and hence $\left\{X_{\chi} \Gamma \Gamma^{\prime} X_{\chi}^{\prime}\right\}^{-1}=\delta I_{\tilde{n}}-\left(\delta^{2} /(1+\delta \tilde{n})\right) \mathbf{1}_{\tilde{n}} \mathbf{1}_{\tilde{n}}^{\prime}$, where $\tilde{n}$ is the number of signals observed under $\chi$.

First, we solve for the equilibrium when all agents choose the same $\chi$. Without loss of generality, suppose that

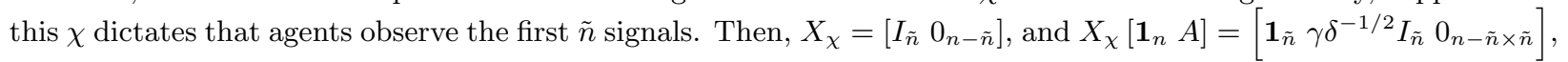
using the fact that $A=\gamma \delta^{-1 / 2} I_{n}$. Therefore,

$$
\begin{aligned}
\bar{\Sigma}(\mu) & =I_{1+n}-\left[\begin{array}{c}
\mathbf{1}_{\tilde{n}}^{\prime} \\
\gamma \delta^{-1 / 2} I_{\tilde{n}} \\
0_{n-\tilde{n} \times \tilde{n}}
\end{array}\right]\left[\delta I_{\tilde{n}}-\left(\delta^{2} /(1+\delta \tilde{n})\right) \mathbf{1}_{\tilde{n}} \mathbf{1}_{\tilde{n}}^{\prime}\right]\left[\mathbf{1}_{\tilde{n}} \gamma \delta^{-1 / 2} I_{\tilde{n}} 0_{n-\tilde{n} \times \tilde{n}}\right] \\
& =I_{1+n}-\left[\begin{array}{ccc}
\frac{\delta \tilde{n}}{1+\delta \tilde{n}} & \frac{\gamma \delta^{1 / 2}}{1+\delta \tilde{n}} \mathbf{1}_{\tilde{n}}^{\prime} & 0_{1 \times n-\tilde{n}} \\
\frac{\gamma \delta^{1 / 2}}{1+\delta \tilde{n}} \mathbf{1}_{\tilde{n}} & \gamma^{2} I_{\tilde{n}}-\frac{\gamma^{2} \delta}{1+\delta \tilde{n}} \mathbf{1}_{\tilde{n}} \mathbf{1}_{\tilde{n}}^{\prime} & 0_{\tilde{n} \times n-\tilde{n}} \\
0_{n-\tilde{n} \times 1} & 0_{n-\tilde{n} \times \tilde{n}} & 0_{n-\tilde{n} \times n-\tilde{n}}
\end{array}\right]
\end{aligned}
$$


Then, $(1-r) I_{1+n}+r \bar{\Sigma}(\mu)$ takes the form

$(1-r) I_{1+n}+r \bar{\Sigma}(\mu)=I_{1+n}-r\left[\begin{array}{ccc}\frac{\delta \tilde{n}}{1+\delta \tilde{n}} & \frac{\gamma \delta^{1 / 2}}{1+\delta \tilde{n}} \mathbf{1}_{\tilde{n}}^{\prime} & 0_{1 \times n-\tilde{n}} \\ \frac{\gamma \delta^{1 / 2}}{1+\delta \tilde{n}} \mathbf{1}_{\tilde{n}} & \gamma^{2} I_{\tilde{n}}-\frac{\gamma^{2} \delta}{1+\delta \tilde{n}} \mathbf{1}_{\tilde{n}} \mathbf{1}_{\tilde{n}}^{\prime} & 0_{\tilde{n} \times n-\tilde{n}} \\ 0_{n-\tilde{n} \times 1} & 0_{n-\tilde{n} \times \tilde{n}} & 0_{n-\tilde{n} \times n-\tilde{n}}\end{array}\right]=\left[\begin{array}{ccc}a & b \mathbf{1}_{\tilde{n}}^{\prime} & 0_{1 \times n-\tilde{n}} \\ b \mathbf{1}_{\tilde{n}} & c I_{\tilde{n}}+d \mathbf{1}_{\tilde{n}} \mathbf{1}_{\tilde{n}}^{\prime} & 0_{\tilde{n} \times n-\tilde{n}} \\ 0_{n-\tilde{n} \times 1} & 0_{n-\tilde{n} \times \tilde{n}} & I_{n-\tilde{n} \times n-\tilde{n}}\end{array}\right]$

where $a=1-r \frac{\delta \tilde{n}}{1+\delta \tilde{n}}, b=-r \frac{\gamma \delta^{1 / 2}}{1+\delta \tilde{n}}, c=1-r \gamma^{2}$, and $d=r \frac{\gamma^{2} \delta}{1+\delta \tilde{n}} .\left[(1-r) I_{1+n}+r \bar{\Sigma}(\mu)\right]^{-1}$ then takes the form

$$
\left[(1-r) I_{1+n}+r \bar{\Sigma}(\mu)\right]^{-1}=\left[\begin{array}{ccc}
\alpha & \beta \mathbf{1}_{\tilde{n}}^{\prime} & 0_{1 \times n-\tilde{n}} \\
\beta \mathbf{1}_{\tilde{n}} & c^{-1} I_{\tilde{n}}+\vartheta \mathbf{1}_{\tilde{n}} \mathbf{1}_{\tilde{n}}^{\prime} & 0_{\tilde{n} \times n-\tilde{n}} \\
0_{n-\tilde{n} \times 1} & 0_{n-\tilde{n} \times \tilde{n}} & I_{n-\tilde{n} \times n-\tilde{n}}
\end{array}\right]
$$

where $a \alpha+\beta b \tilde{n}=1, b \alpha+\beta c+\beta d \tilde{n}=0, a \beta+b / c+b \vartheta \tilde{n}=0$ and $b \beta+c \vartheta+d / c+d \vartheta \tilde{n}=0$. These conditions are solved by

$$
\begin{aligned}
\alpha & =\frac{c+d \tilde{n}}{a(c+d \tilde{n})-b^{2} \tilde{n}}=\frac{1-r \gamma^{2}+\delta \tilde{n}}{1-r \gamma^{2}+(1-r) \delta \tilde{n}} \\
\beta & =-\frac{b}{a(c+d \tilde{n})-b^{2} \tilde{n}}=\frac{r \gamma \delta^{1 / 2}}{1-r \gamma^{2}+(1-r) \delta \tilde{n}} \\
\vartheta & =\frac{\left(b^{2}-a d\right) / c}{a(c+d \tilde{n})-b^{2} \tilde{n}}=\frac{1-r}{1-r \gamma^{2}} \frac{r \gamma^{2} \delta}{1-r \gamma^{2}+(1-r) \delta \tilde{n}}
\end{aligned}
$$

and therefore, $\mathbf{b}^{\prime}\left[(1-r) I_{1+k}+r \bar{\Sigma}(\mu)\right]^{-1}=\left[\alpha \beta \mathbf{1}_{\tilde{n}}^{\prime} 0\right]$.

Next, we derive an agent's best response to the conjectured information choice and equilibrium strategies. Any information choice $\chi$ is summarized by two numbers $\hat{m}$ and $\hat{n}$, where $\hat{n}$ denotes the agents' total number of signals observed, and $\hat{m} \leq \min \{\hat{n}, \tilde{n}\}$ the number of signals that are within the first $\tilde{n}$, and hence shared with all other agents. To confirm our conjecture, we must verify when an agent optimally chooses to observe the same signals as other agents $(\hat{m}=\hat{n}=\tilde{n})$.

Without loss of generality, let us suppose that the agent chooses to observe the first $\hat{m}$ signals (which are shared with all others) and the last $\hat{n}-\hat{m}$ signals (which are not shared). Then,

$$
\begin{aligned}
X_{(\hat{n}, \hat{m})} & =\left[\begin{array}{ccc}
I_{\hat{m}} & 0_{\hat{m} \times n-\hat{n}} & 0_{\hat{m} \times \hat{n}-\hat{m}} \\
0_{\hat{n}-\hat{m} \times \hat{m}} & 0_{\hat{n}-\hat{m} \times n-\hat{n}} & I_{\hat{n}-\hat{m}}
\end{array}\right], \\
X_{(\hat{n}, \hat{m})}\left[\mathbf{1}_{n} A\right] & =\left[\begin{array}{cccc}
\mathbf{1}_{\hat{m}} & \gamma \delta^{-1 / 2} I_{\hat{m}} & 0_{\hat{m} \times n-\hat{n}} & 0_{\hat{m} \times \hat{n}-\hat{m}} \\
\mathbf{1}_{\hat{n}-\hat{m}} & 0_{\hat{n}-\hat{m} \times \hat{m}} & 0_{\hat{n}-\hat{m} \times n-\hat{n}} & \gamma \delta^{-1 / 2} I_{\hat{n}-\hat{m}}
\end{array}\right], \text { and }
\end{aligned}
$$

and $\Sigma(\hat{n}, \hat{m})=I_{1+n}-\left[\mathbf{1}_{n}^{\prime} A^{\prime}\right]^{\prime} X_{(\hat{n}, \hat{m})}^{\prime}\left\{\delta I_{\hat{n}}-\left(\delta^{2} /(1+\delta \hat{n})\right) \mathbf{1}_{\hat{n}} \mathbf{1}_{\hat{n}}^{\prime}\right\} X_{(\hat{n}, \hat{m})}\left[\mathbf{1}_{n} A\right]$, or

$$
\Sigma(\hat{n}, \hat{m})=I_{1+n}-\left[\begin{array}{cccc}
\frac{\hat{n} \delta}{1+\hat{n} \delta} & \frac{\gamma \delta^{1 / 2}}{1+\delta \hat{n}} \mathbf{1}_{\hat{m}}^{\prime} & 0_{1 \times n-\hat{n}} & \frac{\gamma \delta^{1 / 2}}{1+\delta \hat{n}} \mathbf{1}_{\hat{n}-\hat{m}}^{\prime} \\
\frac{\gamma \delta^{1 / 2}}{1+\delta \hat{n}} \mathbf{1}_{\hat{m}} & \gamma^{2} I_{\hat{m}}-\frac{\gamma^{2} \delta}{1+\delta \hat{n}} \mathbf{1}_{\hat{m}} \mathbf{1}_{\hat{m}}^{\prime} & 0_{\hat{m} \times n-\hat{n}} & -\frac{\gamma^{2} \delta}{1+\delta \hat{n}} \mathbf{1}_{\hat{m}} \mathbf{1}_{\hat{n}-\hat{m}}^{\prime} \\
0_{n-\hat{n} \times 1} & 0_{n-\hat{n} \times \hat{m}} & 0_{n-\hat{n} \times n-\hat{n}} & 0_{n-\hat{n} \times \hat{n}-\hat{m}} \\
\frac{\gamma \delta^{1 / 2}}{1+\delta \hat{n}} \mathbf{1}_{\hat{n}-\hat{m}} & -\frac{\gamma^{2} \delta}{1+\delta \hat{n}} \mathbf{1}_{\hat{n}-\hat{m}} \mathbf{1}_{\hat{m}}^{\prime} & 0_{\hat{n}-\hat{m} \times n-\hat{n}} & \gamma^{2} I_{\hat{n}-\hat{m}}-\frac{\gamma^{2} \delta}{1+\delta \hat{n}} \mathbf{1}_{\hat{n}-\hat{m}} \mathbf{1}_{\hat{n}-\hat{m}}^{\prime}
\end{array}\right]
$$

Pre-and post-multiplying this matrix by $\left[\alpha \beta \mathbf{1}_{\tilde{n}}^{\prime} 0\right]$, we find

$$
\begin{aligned}
E U(\hat{n}, \hat{m} ; \tilde{n}) & =-\left\{\alpha^{2}+\tilde{n} \beta^{2}\right\}+\left[\alpha \beta \mathbf{1}_{\hat{m}}^{\prime}\right]\left[\begin{array}{cc}
\frac{\hat{n} \delta}{1+\hat{n} \delta} & \frac{\gamma \delta^{1 / 2}}{1+\delta \hat{n}} \mathbf{1}_{\hat{m}}^{\prime} \\
\frac{\gamma \delta^{1 / 2}}{1+\delta \hat{n}} \mathbf{1}_{\hat{m}} & \gamma^{2} I_{\hat{m}}-\frac{\gamma^{2} \delta}{1+\delta \hat{n}} \mathbf{1}_{\hat{m}} \mathbf{1}_{\hat{m}}^{\prime}
\end{array}\right]\left[\begin{array}{c}
\alpha \\
\beta \mathbf{1}_{\hat{m}}
\end{array}\right] \\
& =-\left\{\alpha^{2}+\tilde{n} \beta^{2}\right\}+\frac{1}{1+\hat{n} \delta}\left\{\hat{n} \delta \alpha^{2}+2 \delta^{1 / 2} \alpha \gamma \hat{m} \beta+\gamma^{2}(1+\delta \hat{n}) \hat{m} \beta^{2}-\gamma^{2} \delta \hat{m}^{2} \beta^{2}\right\} \\
& =-\frac{1}{1+\delta \hat{n}}\left\{\alpha^{2}-2 \delta^{1 / 2} \alpha \gamma \hat{m} \beta+\gamma^{2} \delta \hat{m}^{2} \beta^{2}\right\}-\tilde{n} \beta^{2}+\gamma^{2} \hat{m} \beta^{2} \\
& =-\frac{1}{1+\delta \hat{n}}\left[\alpha-\delta^{1 / 2} \gamma \hat{m} \beta\right]^{2}-\left(\tilde{n}-\gamma^{2} \hat{m}\right) \beta^{2} \\
& =\frac{-1}{\left[1-r \gamma^{2}+(1-r) \delta \tilde{n}\right]^{2}}\left\{r^{2} \gamma^{2}\left(1-\gamma^{2}\right) \delta \tilde{n}+r^{2} \gamma^{4} \delta(\tilde{n}-\hat{m})+\frac{\left[\left(1-r \gamma^{2}\right)(1+\delta \tilde{n})+r \gamma^{2} \delta(\tilde{n}-\hat{m})\right]^{2}}{1+\delta \hat{n}}\right\}
\end{aligned}
$$


When $r \gamma^{2}=0$, this expression reduces to $E U(\hat{n}, \hat{m} ; \tilde{n})=-(1+\delta \tilde{n})^{2}[1+(1-r) \delta \tilde{n}]^{-2}(1+\hat{n} \delta)^{-1}$. In the limit as $\delta \rightarrow 0$, this yields a unique optimal level of information characterized by $\partial E U(\hat{n}, \hat{m} ; \tilde{n}) / \partial \hat{n}=$ $(1+\delta \tilde{n})^{2}[1+(1-r) \delta \tilde{n}]^{-2}(1+\hat{n} \delta)^{-2}=C^{\prime}(\hat{n} \delta)$. At the equilibrium, $\hat{n}=\tilde{n}$, or

$$
\frac{1}{[1+(1-r) \delta \tilde{n}]^{2}}=C^{\prime}(\hat{n} \delta) \text {. }
$$

When $r \gamma^{2}>0, E U(\hat{n}, \hat{m} ; \tilde{n})$ is strictly increasing and concave in $\hat{m}$ for $\hat{m} \leq \tilde{n}$, implying that it is optimal to set $\hat{m}=\min \{\tilde{n}, \hat{n}\}$. We therefore need to check when it is optimal to set $\tilde{n}=\hat{n}$, i.e. acquire exactly the same signals as all the others. For this, we consider separately the two deviations when $\hat{n}>\tilde{n}$ and $\hat{n}<\tilde{n}$. When $\hat{n}>\tilde{n}, \hat{m}=\tilde{n}$, and

$$
E U(\hat{n}, \tilde{n} ; \tilde{n})=-\frac{1}{\left[1-r \gamma^{2}+(1-r) \delta \tilde{n}\right]^{2}}\left\{\frac{\left(1-r \gamma^{2}\right)^{2}(1+\delta \tilde{n})^{2}}{1+\hat{n} \delta}+r^{2} \gamma^{2}\left(1-\gamma^{2}\right) \delta \tilde{n}\right\}
$$

which is increasing and concave in $\hat{n}$. Therefore, $\hat{n}>\tilde{n}$ is strictly worse than $\hat{n}=\tilde{n}$, whenever

$$
\frac{\left(1-r \gamma^{2}\right)^{2}}{\left[1-r \gamma^{2}+(1-r) \delta \tilde{n}\right]^{2}} \leq C^{\prime}(\delta \tilde{n})
$$

On the other hand, if $\hat{n}<\tilde{n}, \hat{m}=\hat{n}$, and

$$
\begin{aligned}
E U(\hat{n}, \hat{n} ; \tilde{n}) & =-\frac{1}{\left[1-r \gamma^{2}+(1-r) \delta \tilde{n}\right]^{2}}\left\{\frac{\left(\left(1-r \gamma^{2}\right)(1+\delta \tilde{n})+r \gamma^{2} \delta(\tilde{n}-\hat{n})\right)^{2}}{1+\hat{n} \delta}+r^{2} \gamma^{2} \delta\left(\tilde{n}-\gamma^{2} \hat{n}\right)\right\} \\
& =-\frac{1}{\left[1-r \gamma^{2}+(1-r) \delta \tilde{n}\right]^{2}}\left\{\left(1-r \gamma^{2}\right)(1+\delta \tilde{n})+r^{2} \gamma^{2}\left(1-\gamma^{2}\right) \delta \tilde{n}+\frac{(1+\delta \tilde{n}) \delta(\tilde{n}-\hat{n})}{1+\hat{n} \delta}\right\}
\end{aligned}
$$

Taking the derivative w.r.t. $\hat{n}$ yields

$$
\frac{d E U(\hat{n}, \hat{n} ; \tilde{n})}{d \hat{n}}=\frac{\delta}{\left[1-r \gamma^{2}+(1-r) \delta \tilde{n}\right]^{2}}\left(\frac{1+\delta \tilde{n}}{1+\delta \hat{n}}\right)^{2}
$$

which is positive and decreasing in $\hat{n}$. Therefore, setting $\hat{n}=\tilde{n}$ is optimal, whenever $\left.(d E U(\hat{n}, \hat{n} ; \tilde{n}) / d \hat{n})\right|_{\hat{n}=\tilde{n}} \geq$ $\delta C^{\prime}(\delta \tilde{n})$, or equivalently,

$$
\frac{1}{\left[1-r \gamma^{2}+(1-r) \delta \tilde{n}\right]^{2}} \geq C^{\prime}(\delta \tilde{n})
$$

Finally, when $r \gamma^{2}<0$, we wish to rule out the existence of pure strategy equilibria. To do so, we consider the change in payoffs of an agent who deviates from the proposed equilibrium profile of setting $\tilde{n}=\hat{n}=\hat{m}$, by increasing $\hat{n}$ to $\tilde{n}+1$, or by reducing $\hat{n}$ and $\hat{m}$ to $\tilde{n}-1$. The above derivations for the case $r \gamma^{2}>0$ imply that the former deviation increases payoffs, whenever $\left(1-r \gamma^{2}\right)^{2} /\left[1-r \gamma^{2}+(1-r) \delta \tilde{n}\right]^{2}>C^{\prime}(\delta \tilde{n})$, while the latter increases payoffs, whenever $1 /\left[1-r \gamma^{2}+(1-r) \delta \tilde{n}\right]^{2}<C^{\prime}(\delta \tilde{n})$. When $r \gamma^{2}<0$, we immediately find that either one or the other of these inequalities is satisfied, thus ruling out the possibility of a pure strategy equilibrium.

\section{Uniqueness of Private information equilibrium}

The above proof implies that there is a unique pure strategy equilibrium in the limit as $\delta \rightarrow 0$, when information is private, or when there are no complementarities $\left(r \gamma^{2}=0\right)$. To show that this is the unique equilibrium, we need to rule out the possibility of mixed equilibria in the limit. Now, notice that when $A=0$,

$$
\Sigma(\chi)=\left(\begin{array}{cc}
\Sigma_{11}(\chi) & 0_{1 \times n} \\
0_{n \times 1} & I_{n}
\end{array}\right)
$$

where the $(1,1)$ th entry $\Sigma_{11}(\chi)$ is given by $\Sigma_{11}(\chi)=1-\mathbf{1}_{n}^{\prime} X_{\chi}^{\prime}\left\{X_{\chi} \Gamma \Gamma^{\prime} X_{\chi}^{\prime}\right\}^{-1} X_{\chi} \mathbf{1}_{n}=\left(1+\delta \tilde{n}_{\chi}\right)^{-1}$, where $\tilde{n}_{\chi}$ is the number of signals observed under $\chi$. Payoffs in turn are given by $E U(\chi, \mu)=\left[1-r+r \bar{\Sigma}_{11}(\mu)\right]^{-2} \Sigma_{11}(\chi)$, where $\bar{\Sigma}_{11}(\mu)$ is the $(1,1)$ th entry of $\bar{\Sigma}(\mu)$. In this special case, beliefs and actions are not affected by common signal noise, and hence payoffs are only affected by individual and average uncertainty about the state $s$, which is measured by the $(1,1)$ entries of $\Sigma_{11}(\chi)$ and $\bar{\Sigma}_{11}(\mu)$. Weakening the requirement of definitions 1 and 2), we can say that information choice $\chi$ is more informative as $\chi^{\prime}$, if and only if $\tilde{n}_{\chi} \geq \tilde{n}_{\chi^{\prime}}$.

Since $\Sigma_{11}(\chi)$ is decreasing and convex in the number of observed signals $\tilde{n}_{\chi}$, there exists a unique optimal precision level $\phi^{*}(\bar{\Sigma})$ that minimizes $\left[1-r+r \bar{\Sigma}_{11}(\mu)\right]^{-2}\left(1+\phi^{*}\right)^{-1}-C\left(\phi^{*}\right)$, for any $\bar{\Sigma} \in[0,1]$. Thus for any 
aggregate information choice distribution $\mu$, and resulting $\bar{\Sigma}_{11}(\mu)$, the agent's optimal information choice is almost everywhere unique, and is no more than $\delta$ away from $\phi^{*}\left(\bar{\Sigma}_{11}(\mu)\right)$. In the limit as $\delta \rightarrow 0$, the agent's best response profile is therefore characterized by $\phi^{*}\left(\bar{\Sigma}_{11}(\mu)\right)$, which rules out the possibility of mixed strategies being optimal in the limit.

\section{A.6 Proof of Lemma 2: Equilibrium price in the planning model}

Conjecture that $p(t)$ takes the form $p(t)=\sigma \int_{-\infty}^{t} g_{t}(\tau) d Z(\tau)$ for some function $g_{t}(\tau)$. Then, $p^{*}(t)=\sigma$.

$\int_{-\infty}^{t}\left(1-r+r g_{t}(\tau)\right) d Z(\tau)$ and $E\left(p^{*}(t) \mid I_{\hat{\tau}}\right)=\sigma \int_{-\infty}^{\hat{\tau}}\left(1-r+r g_{t}(\tau)\right) d Z(\tau)$. Since $p(t)=\int_{-\infty}^{t} E\left(p^{*}(t) \mid I_{\hat{\tau}}\right) d \Lambda_{t}(\hat{\tau})$,

$$
\begin{aligned}
p(t) & =\sigma \int_{-\infty}^{t} \int_{-\infty}^{\hat{\tau}}\left(1-r+r g_{t}(\tau)\right) d Z(\tau) d \Lambda_{t}(\hat{\tau}) \\
& =\left.\sigma \Lambda_{t}(\hat{\tau}) \int_{-\infty}^{\hat{\tau}}\left(1-r+r g_{t}(\tau)\right) d Z(\tau)\right|_{\hat{\tau}=-\infty} ^{t}-\sigma \int_{-\infty}^{t} \Lambda_{t}(\hat{\tau})\left(1-r+r g_{t}(\hat{\tau})\right) d Z(\hat{\tau}) \\
& =\sigma \int_{-\infty}^{t}\left(1-\Lambda_{t}(\tau)\right)\left(1-r+r g_{t}(\tau)\right) d Z(\tau)
\end{aligned}
$$

where the last step follows from changing the order of integration. Setting $g_{t}(\tau)=\left(1-\Lambda_{t}(\tau)\right)\left(1-r+r g_{t}(\tau)\right)$ yields the result.

\section{A.7 Proof of Proposition 3: Staggered planning equilibria}

Suppose that all other firms plan every $T$ periods, and the proportion of firms planning over any interval of length $d t$ is $T^{-1} d t$. Clearly, $\left\{\Lambda_{t}(\tau)\right\}$ and $L(t, \hat{\tau})$ only depend on the time $\delta=t-\hat{\tau}$ since the last planning date, and are stationary over time w.r.t. $\delta$. Using $\delta$ as a state variable, the Bellman equation can now be rewritten as

$$
\mathcal{L}(\delta)=\min _{\hat{\delta} \geq \delta}\left\{\int_{0}^{\hat{\delta}-\delta} e^{-\rho \delta^{\prime}} L\left(\delta^{\prime}\right) d \delta^{\prime}+e^{-\rho(\hat{\delta}-\delta)}[C+\mathcal{L}(0)]\right\}
$$

where the loss function is

$$
L(\delta)=\left\{\begin{array}{c}
\sigma^{2} \int_{0}^{\delta} \frac{(1-r)^{2}}{\left(1-r \frac{\delta^{\prime}}{T}\right)^{2}} d \delta^{\prime} \text { if } \delta \leq T \\
\sigma^{2} \int_{0}^{T} \frac{(1-r)^{2}}{\left(1-r \frac{\delta^{\prime}}{T}\right)^{2}} d \delta^{\prime}+\sigma^{2}(\delta-T) \text { if } \delta>T
\end{array}\right.
$$

The solution to this Bellman equation is characterized by an optimal horizon $T^{*}$, s.t. it is optimal to plan at date $t$, if and only if $t \geq s+T^{*}$. $T^{*}$ solves the first-order condition $L\left(T^{*}\right)=\rho[C+\mathcal{L}(0)]$, where $\mathcal{L}(0)=$ $\left(\int_{0}^{T^{*}} e^{-\rho \delta} L(\delta) d \delta+e^{-\rho T^{*}} C\right) /\left(1-e^{-\rho T^{*}}\right)$. In equilibrium, $T^{*}=T$, so $T^{*}$ has to satisfy

$$
L\left(T^{*}\right)=\frac{\rho}{1-e^{-\rho T^{*}}}\left[\int_{0}^{T^{*}} e^{-\rho \delta} L(\delta) d \delta+C\right]
$$

or $C=\int_{0}^{T^{*}} e^{-\rho \delta}\left[L\left(T^{*}\right)-L(\delta)\right] d \delta$. Changing variables to $\theta=\frac{\delta}{T^{*}}$, we write $L(\delta)$ as

$$
L(\delta)=\sigma^{2} T^{*} \int_{0}^{\delta / T^{*}} \frac{(1-r)^{2}}{(1-r \theta)^{2}} d \theta=\sigma^{2} T^{*} \frac{(1-r)^{2}}{r}\left(\frac{1}{1-r \delta / T^{*}}-1\right)=\sigma^{2}(1-r)^{2} T^{*} \frac{\delta}{T^{*}-r \delta},
$$

with $L\left(T^{*}\right)=(1-r) T^{*}$. Substituting into the equilibrium condition, we have $L\left(T^{*}\right)-L(\delta)$ $=(1-r) T^{*}\left(T^{*}-\delta\right) /\left(T^{*}-r \delta\right)$, and therefore

$$
C=\sigma^{2}(1-r) T^{*} \int_{0}^{T^{*}} e^{-\rho \delta} \frac{T^{*}-\delta}{T^{*}-r \delta} d \delta
$$

The right side of this expression is increasing in $T^{*}$, because $r<1$. Thus, there exists a unique solution to this equilibrium condition, which is increasing in $C$. With a change of variables, we can rewrite this equation as

$$
C=\sigma^{2}(1-r)\left(T^{*}\right)^{2} \int_{0}^{1} e^{-\rho T^{*} \theta} \frac{1-\theta}{1-r \theta} d \theta
$$


This formulation reveals that the equilibrium planning horizon $T$ is increasing in $r$ and $\rho$ because $e^{-\rho \tau} \frac{T-\tau}{T-r \tau}(1-r)$ is decreasing in $r$ and in $\rho$ for all $\tau \in(0, T)$. Taking the limit as $\rho \rightarrow 0$, the last expression converges to $\sigma^{2}\left(T^{*}\right)^{2} g(r)$, with

$$
g(r)=\int_{0}^{1} \frac{(1-\theta)(1-r)}{1-r \theta} d \theta=\frac{1-r}{r}\left\{1+\frac{1-r}{r} \log (1-r)\right\} .
$$

\section{A.8 Proof of Proposition 4: Existence of synchronized planning equilibrium}

For arbitrary $T>0$ let $\mathcal{T}^{*}=\{n T\}_{n \in \mathbb{N}}$ denote the conjectured equilibrium strategy, for a synchronized planning equilibrium with horizon $T$. We begin by defining the expected loss $\mathcal{L}(\mathcal{T})$ that is associated with an arbitrary sequence of planning dates, $\mathcal{T}=\left\{\hat{\tau}_{n}\right\}_{n \in \mathbb{N}}$, when all other firms use strategy $\mathcal{T}^{*}$. $\mathcal{T}^{*}$ constitutes a synchronized planning equilibrium, if and only if $\Delta(\mathcal{T}) \equiv \mathcal{L}(\mathcal{T})-\mathcal{L}\left(\mathcal{T}^{*}\right) \geq 0$, for all sequences $\mathcal{T}$.

Under $\mathcal{T}^{*}$, the instantaneous loss function at date $t$ is $L^{*}(t)=(1-r)^{2} \sigma^{2}(t-\operatorname{Tint}(t / T))$, where int $(x)$ denotes the integer part of a real number $x$. Subtracting it from $L(t, \hat{\tau})$, we have

$$
L(t, \hat{\tau})-L^{*}(t)=\left\{\begin{array}{c}
(1-r)^{2} \sigma^{2}(\operatorname{Tint}(t / T)-\hat{\tau}) \text { if } \hat{\tau} \geq \operatorname{Tint}(t / T) \\
\sigma^{2}(\operatorname{Tint}(t / T)-\hat{\tau}) \text { if } \hat{\tau}<\operatorname{Tint}(t / T) .
\end{array}\right.
$$

We can rewrite $L(t, \hat{\tau})-L^{*}(t)$ in two different ways, that will be useful below:

$$
\begin{aligned}
L(t, \hat{\tau})-L^{*}(t) & =(1-r)^{2} \sigma^{2}(\operatorname{Tint}(t / T)-\hat{\tau})+\sigma^{2}\left(1-(1-r)^{2}\right) \max \{0, \operatorname{Tint}(t / T)-\hat{\tau}\} \\
\text { or } L(t, \hat{\tau})-L^{*}(t) & =\sigma^{2}(\operatorname{Tint}(t / T)-\hat{\tau})-\sigma^{2}\left(1-(1-r)^{2}\right) \min \{0, \operatorname{Tint}(t / T)-\hat{\tau}\} \\
& =\sigma^{2}(\operatorname{Tint}(t / T)-\hat{\tau})+\sigma^{2}\left(1-(1-r)^{2}\right) \max \{0, \hat{\tau}-\operatorname{Tint}(t / T)\}
\end{aligned}
$$

A convex combination of the above expressions, for any $\lambda \in[0,1]$, gives

$$
\begin{aligned}
L(t, \hat{\tau})-L^{*}(t)= & \sigma^{2}\left[\lambda(1-r)^{2}+1-\lambda\right](\operatorname{Tint}(t / T)-\hat{\tau}) \\
& +\sigma^{2}\left(1-(1-r)^{2}\right)[\lambda \max \{0, \operatorname{Tint}(t / T)-\hat{\tau}\}+(1-\lambda) \max \{0, \hat{\tau}-\operatorname{Tint}(t / T)\}]
\end{aligned}
$$

Now, $\Delta(\mathcal{T})$ is given by:

$$
\begin{aligned}
\Delta(\mathcal{T})= & \sigma^{2} \sum_{n=0}^{\infty} \int_{\hat{\tau}_{n}}^{\hat{\tau}_{n+1}} e^{-\rho t}\left[\lambda(1-r)^{2}+1-\lambda\right]\left(\operatorname{Tint}(t / T)-\hat{\tau}_{n}\right) d t+\sum_{n=0}^{\infty}\left(e^{-\rho \hat{\tau}_{n}}-e^{-\rho n T}\right) C \\
& +\sigma^{2}\left(1-(1-r)^{2}\right) \sum_{n=0}^{\infty} \int_{\hat{\tau}_{n}}^{\hat{\tau}_{n+1}} e^{-\rho t}\left[\lambda \max \left\{0, \operatorname{Tint}(t / T)-\hat{\tau}_{n}\right\}+(1-\lambda) \max \left\{0, \hat{\tau}_{n}-\operatorname{Tint}(t / T)\right\}\right] d t
\end{aligned}
$$

where $\hat{\tau}_{0}=0$. Solving the integral terms in the first line gives

$$
\begin{aligned}
\sum_{n=0}^{\infty} \int_{\hat{\tau}_{n}}^{\hat{\tau}_{n+1}} e^{-\rho t} \operatorname{Tint}(t / T) d t & =\int_{0}^{\infty} e^{-\rho t} \operatorname{Tint}(t / T) d t=\sum_{n=0}^{\infty} n T e^{-\rho n T} \int_{0}^{T} e^{-\rho t} d t=\frac{1}{\rho} T \frac{e^{-\rho T}}{1-e^{-\rho T}} \\
\sum_{n=0}^{\infty} \int_{\hat{\tau}_{n}}^{\hat{\tau}_{n+1}} e^{-\rho t} \hat{\tau}_{n} d t & =\frac{1}{\rho} \sum_{n=0}^{\infty} \hat{\tau}_{n}\left[e^{-\rho \hat{\tau}_{n}}-e^{-\rho \hat{\tau}_{n+1}}\right]=\frac{1}{\rho} \sum_{n=1}^{\infty} e^{-\rho \hat{\tau}_{n}}\left(\hat{\tau}_{n}-\hat{\tau}_{n-1}\right) \\
\sum_{n=0}^{\infty} e^{-\rho n T} C & =\frac{1}{1-e^{-\rho T}} C
\end{aligned}
$$

and therefore, $\Delta(\mathcal{T})$ can be rewritten as

$$
\begin{aligned}
\Delta(\mathcal{T})= & \frac{1}{\rho} \frac{e^{-\rho T}}{1-e^{-\rho T}}\left\{\sigma^{2}\left[1-\lambda+\lambda(1-r)^{2}\right] T-\rho C\right\}-\frac{1}{\rho} \sum_{n=1}^{\infty} e^{-\rho \hat{\tau}_{n}}\left\{\sigma^{2}\left[1-\lambda+\lambda(1-r)^{2}\right]\left(\hat{\tau}_{n}-\hat{\tau}_{n-1}\right)-\rho C\right\} \\
& +\sigma^{2}\left(1-(1-r)^{2}\right) \sum_{n=0}^{\infty} \int_{\hat{\tau}_{n}}^{\hat{\tau}_{n+1}} e^{-\rho t}\left[\lambda \max \left\{0, \operatorname{Tint}(t / T)-\hat{\tau}_{n}\right\}+(1-\lambda) \max \left\{0, \hat{\tau}_{n}-\operatorname{Tint}(t / T)\right\}\right] d t
\end{aligned}
$$

Case $(i)(r \in(0,1))$ : For all $\lambda \in[0,1]$, the second line of this expression is non-negative, and it is zero if and only if $\mathcal{T}=\mathcal{T}^{*}$ Therefore, if, for some $\lambda \in[0,1], \mathcal{T}^{*}$ also minimizes the first line of this expression, it must be the 
case that $\Delta(\mathcal{T})$ is minimized at $\mathcal{T}^{*}$, and hence that $\mathcal{T}^{*}$ constitutes an equilibrium. Taking first-order conditions, the first line is minimized, if and only if $\rho C=\left[1-\lambda+\lambda(1-r)^{2}\right]\left(\hat{\tau}_{n}-\hat{\tau}_{n-1}-\frac{1}{\rho}\left(1-e^{-\rho\left(\hat{\tau}_{n+1}-\hat{\tau}_{n}\right)}\right)\right)$, and $\mathcal{T}^{*}$ satisfies these first order conditions, if and only if

$$
\rho C=\sigma^{2}\left[1-\lambda+\lambda(1-r)^{2}\right]\left(T-\frac{1}{\rho}\left(1-e^{-\rho T}\right)\right)
$$

Moreover, we have

$T-\frac{1}{\rho}\left(1-e^{-\rho T}\right)=\int_{0}^{T}\left[1-e^{-\rho t}\right] d t=\left[1-e^{-\rho T}\right] T-\rho \int_{0}^{T} e^{-\rho t} t d t=\rho \int_{0}^{T} e^{-\rho t}(T-t) d t=\rho T^{2} \int_{0}^{1} e^{-\rho T \theta}(1-\theta) d \theta$.

Therefore, the first-order condition is satisfied for $\underline{T}$ at $\lambda=0$, for $\bar{T}$ at $\lambda=1$, and for every $T \in[\underline{T}, \bar{T}]$ at some intermediary $\lambda \in[0,1]$. We conclude that any $T \in[\underline{T}, \bar{T}]$ is sustainable as a synchronized equilibrium planning horizon.

Case $(i i)(r=0)$ : the second line in our expression for $\Delta(\mathcal{T})$ above is exactly equal to zero, so minimizing the first line is also a necessary condition for $\mathcal{T}^{*}$ to be an equilibrium. Moreover, in this case, we have $\underline{T}=\bar{T}=T^{*}$, so there exists a unique synchronized equilibrium. Moreover, notice that the horizon in this case is identical to the staggered planning equilibrium. Since there is no interaction in pricing and planning decisions, the distribution of planning choices is indeterminate in equilibrium.

Case $($ iii $)(r<0)$ : now, the second line in the expression for $\Delta(\mathcal{T})$ is negative, and is zero only if $\mathcal{T}=\mathcal{T}^{*}$. Consider then a firm that sets $\hat{\tau}_{1}=T+\delta$ (for small $\delta>0$ ), but keeps all other planning dates unchanged from $\mathcal{T}^{*}$, setting $\hat{\tau}_{n}=n T$. The resulting change in expected loss is:

$$
\begin{aligned}
\Delta_{+} & =\frac{1}{\rho} e^{-\rho T}\left\{\left[1-e^{-\rho \delta}\right]\left(\sigma^{2} T-\rho C\right)-(1-r)^{2} \sigma^{2} \delta\left[e^{-\rho \delta}-e^{-\rho T}\right]\right\} \\
\text { with limit } \lim _{\delta \rightarrow 0}\left(\Delta_{+} / \delta\right) & =e^{-\rho T}\left\{\sigma^{2} T-\rho C-(1-r)^{2} \sigma^{2} \frac{1}{\rho}\left[1-e^{-\rho T}\right]\right\}
\end{aligned}
$$

If instead, the firm sets $\hat{\tau}_{1}=T-\delta$ for small $\delta$, but keeps all other planning dates unchanged, the resulting change in expected loss is

$$
\begin{aligned}
\Delta_{-} & =\frac{1}{\rho} e^{-\rho T}\left\{\sigma^{2} \delta\left[1-e^{-\rho T}\right]-\left[e^{\rho \delta}-1\right]\left((1-r)^{2} \sigma^{2}(T-\delta)-\rho C\right)\right\} \\
\text { with limit } \lim _{\delta \rightarrow 0}\left(\Delta_{-} / \delta\right) & =e^{-\rho T}\left\{\sigma^{2} \frac{1}{\rho}\left[1-e^{-\rho T}\right]-\left((1-r)^{2} \sigma^{2} T-\rho C\right)\right\}
\end{aligned}
$$

$\mathcal{T}^{*}$ can be an equilibrium only if $\lim _{\delta \rightarrow 0}\left(\Delta_{+} / \delta\right) \geq 0$ and $\lim _{\delta \rightarrow 0}\left(\Delta_{-} / \delta\right) \geq 0$, but the above immediately implies that

$$
\begin{aligned}
\lim _{\delta \rightarrow 0}\left(\Delta_{+} / \delta\right)+\lim _{\delta \rightarrow 0}\left(\Delta_{-} / \delta\right)= & e^{-\rho T}\left\{\sigma^{2} T-\rho C-(1-r)^{2} \sigma^{2} \frac{1}{\rho}\left[1-e^{-\rho T}\right]\right\} \\
& +e^{-\rho T}\left\{\sigma^{2} \frac{1}{\rho}\left[1-e^{-\rho T}\right]-\left((1-r)^{2} \sigma^{2} T-\rho C\right)\right\} \\
= & e^{-\rho T} \sigma^{2}\left(1-(1-r)^{2}\right)\left\{T+\frac{1}{\rho}\left[1-e^{-\rho T}\right]\right\}<0,
\end{aligned}
$$

implying that either $\lim _{\delta \rightarrow 0}\left(\Delta_{+} / \delta\right)<0$ or $\lim _{\delta \rightarrow 0}\left(\Delta_{-} / \delta\right)<0$, and contradicting the possibility of a synchronized equilibrium.

\section{A.9 Necessary conditions for synchronized planning}

Here, we construct bounds $\underline{\underline{T}}$ and $\overline{\bar{T}}$, such that a synchronized planning equilibrium only exists for planning horizons $T \in[\underline{\underline{T}}, \overline{\bar{T}}]$. Using the numerical procedure discussed in the main text, we check that these conditions are also sufficient.

Suppose that all other firms plan at dates $\{T, 2 T, \ldots\}$. We construct these bounds by showing that whenever $T \notin[\underline{\underline{T}}, \overline{\bar{T}}]$, the agent prefers to deviate from the proposed sequence of planning dates $\{T, 2 T, \ldots\}$, in one of four ways: $(i)$ adding some additional planning date $T^{\prime}$ to the sequence, $(i i)$ skipping a planning date $n T$ for some $n$, 
(iii) delaying a planning date from $n T$ to $n T+\Delta$ (where $\Delta$ is infinitesimal), and (iv) moving forward a planning date from $n T$ to $n T-\Delta$ (where $\Delta$ is again infinitesimal). We now provide conditions on $T$ under which these four deviations are not desirable.

(i) adding a planning date $T^{\prime}$, between between $T$ and $2 T$ : This deviation reduces expected flow losses by $(1-r)^{2} \sigma^{2}\left(T^{\prime}-T\right)$ between dates $T^{\prime}$ and $2 T$, but incurs an additional planning cost at date $T^{\prime}$. Combining these terms, the deviation is not profitable, whenever $C \geq(1-r)^{2} \sigma^{2} \int_{T^{\prime}}^{2 T}\left(T^{\prime}-T\right) e^{-\rho\left(t-T^{\prime}\right)} d t$. Since this must hold for every $T^{\prime} \in[T, 2 T]$, the condition can be rewritten as $C \geq(1-r)^{2} \sigma^{2} \min _{x \in[0, T]} x \int_{x}^{T} e^{-\rho(t-x)} d t$

$=(1-r)^{2} \sigma^{2} \min _{x \in[0, T]} x\left(1-e^{-\rho(T-x)}\right) / \rho$.

(ii) skipping a planning date $n T$ : This deviation saves the planning cost $C$ at date $n T$, but increases the expected losses by $\sigma^{2} T$ from $n T$ to $(n+1) T$, so the deviation is not profitable, whenever $C \leq \sigma^{2} T \int_{0}^{T} e^{-\rho t} d t=$ $\sigma^{2} T\left(1-e^{-\rho T}\right) / \rho$.

Combining these two conditions, we have

$$
(1-r)^{2} \sigma^{2} \min _{x \in[0, T]} x \frac{1-e^{-\rho(T-x)}}{\rho} \leq C \leq \sigma^{2} T \frac{1-e^{-\rho T}}{\rho}
$$

which implicitly defines $\underline{\underline{T}}_{1}$ and $\overline{\bar{T}}_{1}$. As $\rho \rightarrow 0$, this condition converges to $(1-r)^{2} \sigma^{2} T^{2} / 4 \leq C \leq \sigma^{2} T^{2}$, or $\underline{\underline{T}}_{1}=\sqrt{C} / \sigma$, and $\overline{\bar{T}}_{1}=2 /(1-r) \cdot \sqrt{C} / \sigma$. Clearly, this spans the set $[\underline{T}, \bar{T}]$.

In addition, we check that no infinitesimal deviation from the proposed planning dates can be optimal.

(iii) delaying a planning date from $n T$ to $n T+\Delta$ : This deviation postpones the planning cost, and reduces expected losses between $n T+\Delta$ and $(n+1) T$ by $(1-r)^{2} \Delta \sigma^{2}$, but it increases expected losses between $n T$ and $n T+\Delta$ by $T \sigma^{2}$. Combining these terms, the deviation is not profitable, whenever

$$
C\left[1-e^{-\rho \Delta}\right]+\Delta(1-r)^{2} \sigma^{2} \int_{\Delta}^{T} e^{-\rho t} d t \leq T \sigma^{2} \int_{0}^{\Delta} e^{-\rho t} d t
$$

Letting $\Delta \rightarrow 0$, the condition becomes $\rho C \leq \sigma^{2}\left(T-(1-r)^{2}\left(1-e^{-\rho T}\right) / \rho\right)$, which defines an additional lower bound $\underline{T}_{2}$.

(iv) advancing a planning date from $n T$ to $n T-\Delta$ : This deviation advances the planning cost by $\Delta$, and increases expected losses between $n T$ and $T+1$ by $\Delta \sigma^{2}$, but lowers expected losses between $n T$ and $n T-\Delta$ by $(1-r)^{2}(T-\Delta) \sigma^{2}$. Combining these terms, the deviation is not profitable, whenever

$$
C\left[e^{\rho \Delta}-1\right]+\Delta \sigma^{2} \int_{0}^{T} e^{-\rho t} d t \geq(1-r)^{2}(T-\Delta) \sigma^{2} \int_{0}^{\Delta} e^{\rho t} d t
$$

Letting $\Delta \rightarrow 0$, the condition becomes $\rho C \geq \sigma^{2}\left((1-r)^{2} T-\frac{1}{\rho}\left[1-e^{-\rho T}\right]\right)$, which defines an additional upper bound $\overline{\bar{T}}_{2}$.

A synchronized planning equilibrium therefore only exists for $T \in[\underline{\underline{T}}, \overline{\bar{T}}]$, where $\underline{\underline{T}}=\max \left\{\underline{\underline{T}}_{1}, \underline{\underline{T}}_{2}\right\}$ and $\overline{\bar{T}}=$ $\min \left\{\overline{\bar{T}}_{1}, \overline{\bar{T}}_{2}\right\}$. As $\rho \rightarrow 0, \underline{\underline{T}}_{2} \rightarrow 0$ and $\overline{\bar{T}}_{2} \rightarrow \infty$, so the first set of bounds becomes the relevant one. 